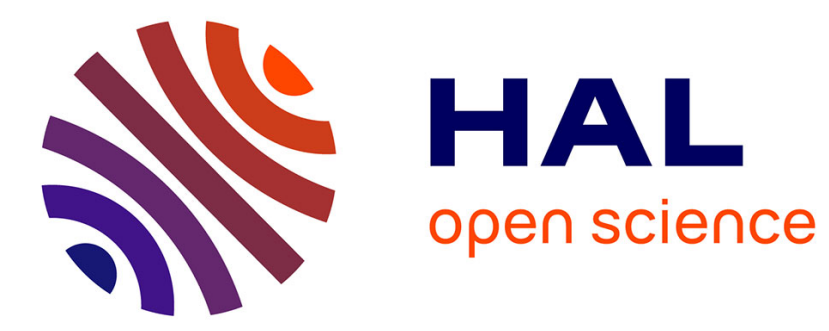

\title{
The Periodic Unfolding Method in Homogenization
}

\author{
Doina Cioranescu, Alain Damlamian, Georges Griso
}

\section{To cite this version:}

Doina Cioranescu, Alain Damlamian, Georges Griso. The Periodic Unfolding Method in Homogenization. SIAM Journal on Mathematical Analysis, 2008, 40 (4), pp.1585-1620. 10.1137/080713148 . hal-00693080

\section{HAL Id: hal-00693080 \\ https://hal.science/hal-00693080}

Submitted on 1 May 2012

HAL is a multi-disciplinary open access archive for the deposit and dissemination of scientific research documents, whether they are published or not. The documents may come from teaching and research institutions in France or abroad, or from public or private research centers.
L'archive ouverte pluridisciplinaire HAL, est destinée au dépôt et à la diffusion de documents scientifiques de niveau recherche, publiés ou non, émanant des établissements d'enseignement et de recherche français ou étrangers, des laboratoires publics ou privés. 


\title{
THE PERIODIC UNFOLDING METHOD IN HOMOGENIZATION
}

\author{
D. CIORANESCU ${ }^{\dagger}$, A. DAMLAMIAN ${ }^{\ddagger}$, AND G. GRISO $§$
}

\begin{abstract}
The periodic unfolding method was introduced in 2002 in [Cioranescu, Damlamian, and Griso, C.R. Acad. Sci. Paris, Ser. 1, 335 (2002), pp. 99-104] (with the basic proofs in [Proceedings of the Narvik Conference 2004, GAKUTO Internat. Ser. Math. Sci. Appl. 24, Gakkōtosho, Tokyo, 2006, pp. 119-136]). In the present paper we go into all the details of the method and include complete proofs, as well as several new extensions and developments. This approach is based on two distinct ideas, each leading to a new ingredient. The first idea is the change of scale, which is embodied in the unfolding operator. At the expense of doubling the dimension, this allows one to use standard weak or strong convergence theorems in $L^{p}$ spaces instead of more complicated tools (such as two-scale convergence, which is shown to be merely the weak convergence of the unfolding; cf. Remark 2.15). The second idea is the separation of scales, which is implemented as a macro-micro decomposition of functions and is especially suited for the weakly convergent sequences of Sobolev spaces. In the framework of this method, the proofs of most periodic homogenization results are elementary. The unfolding is particularly well-suited for multiscale problems (a simple backward iteration argument suffices) and for precise corrector results without extra regularity on the data. A list of the papers where these ideas appeared, at least in some preliminary form, is given with a discussion of their content. We also give a list of papers published since the publication [Cioranescu, Damlamian, and Griso, C.R. Acad. Sci. Paris, Ser. 1, 335 (2002), pp. 99-104], and where the unfolding method has been successfully applied.
\end{abstract}

Key words. homogenization, periodic unfolding, multiscale problems

1. Introduction. The notion of two-scale convergence was introduced in 1989 by Nguetseng in [58], further developed by Allaire in [1] and by Lukkassen, Nguetseng, and Wall in [55] with applications to periodic homogenization. It was generalized to some multiscale problems by Ene and Saint Jean Paulin in [38], Allaire and Briane in [2], Lions et al. in [52] and Lukkassen, Nguetseng, and Wall in [55].

In 1990, Arbogast, Douglas, and Hornung defined a "dilation" operator in [5] to study homogenization for a periodic medium with double porosity. This technique was used again in [16], [3], [4], [48], [49], [50], [51], [54], [20], [21], [22], and [23].

In [24], we expanded on this idea and presented a general and quite simple approach for classical or multiscale periodic homogenization, under the name of "unfolding method." Originally restricted to the case of domains consisting of a union of $\varepsilon$-cells, it was extended to general domains (see the survey of Damlamian [34]). In the present work, we give a complete presentation of this method, including all of the proofs, as well as several new extensions and developments. The relationship of the papers listed above with our work is discussed at the end of this introduction.

The periodic unfolding method is essentially based on two ingredients. The first one is the unfolding operator $\mathcal{T}_{\varepsilon}$ (similar to the dilation operator), defined in section 2 ,

\footnotetext{
${ }^{\dagger}$ Corresponding author. Laboratoire Jacques-Louis Lions, Université Pierre et Marie Curie, Boîte courrier 187, 4 Place Jussieu, 75252 Paris Cedex 05, France (cioran@ann.jussieu.fr).

‡Université Paris-Est, Laboratoire d'Analyse et de Mathématiques Appliquées, CNRS UMR 8050, Centre Multidisciplinaire de Créteil, 94010, Créteil, Cedex, France (damla@univ-paris12.fr).

$\S$ Corresponding author. Laboratoire Jacques-Louis Lions, Université Pierre et Marie Curie, Boîte courrier 187, 4 Place Jussieu, 75252 Paris Cedex 05, France (griso@ann.jussieu.fr).
} 
where its properties are investigated. Let $\Omega$ be a bounded open set, and $Y$ a reference cell in $\mathbb{R}^{n}$. By definition, the operator $\mathcal{T}_{\varepsilon}$ associates to any function $v$ in $L^{p}(\Omega)$, a function $\mathcal{T}_{\varepsilon}(v)$ in $L^{p}(\Omega \times Y)$. An immediate (and interesting) property of $\mathcal{T}_{\varepsilon}$ is that it enables one to transform any integral over $\Omega$ in an integral over $\Omega \times Y$. Indeed, by Proposition 2.6 below

$$
\int_{\Omega} w(x) d x \sim \frac{1}{|Y|} \int_{\Omega \times Y} \mathcal{T}_{\varepsilon}(w)(x, y) d x d y \quad \forall w \in L^{1}(\Omega) .
$$

Proposition 2.14 shows that the two-scale convergence in the $L^{p}(\Omega)$-sense of a sequence of functions $\left\{v_{\varepsilon}\right\}$ is equivalent to the weak convergence of the sequence of unfolded functions $\left\{\mathcal{T}_{\varepsilon}\left(v_{\varepsilon}\right)\right\}$ in $L^{p}(\Omega \times Y)$. Thus, the two-scale convergence in $\Omega$ is reduced to a mere weak convergence in $L^{p}(\Omega \times Y)$, which conceptually simplifies proofs.

In section 2 are also introduced a local average operator $\mathcal{M}_{\varepsilon}$ and an averaging operator $\mathcal{U}_{\varepsilon}$, the latter being, in some sense, the inverse of the unfolding operator $\mathcal{T}_{\varepsilon}$.

The second ingredient of the periodic unfolding method consists of separating the characteristic scales by decomposing every function $\varphi$ belonging to $W^{1, p}(\Omega)$ in two parts. In section 3 it is achieved by using the local average. In section 4 , the original proof of this scale-splitting, inspired by the finite element method (FEM), is given. The confrontation of the two methods of sections 3 and 4 is interesting in itself (Theorem 3.5 and Proposition 4.8). In both approaches, $\varphi$ is written as $\varphi=\varphi_{1}^{\varepsilon}+\varepsilon \varphi_{2}^{\varepsilon}$, where $\varphi_{1}^{\varepsilon}$ is a macroscopic part designed not to capture the oscillations of order $\varepsilon$ (if there are any), while the microscopic part $\varphi_{2}^{\varepsilon}$ is designed to do so. The main result states that, from any bounded sequence $\left\{w^{\varepsilon}\right\}$ in $W^{1, p}(\Omega)$, weakly convergent to some $w$, one can always extract a subsequence (still denoted $\left\{w^{\varepsilon}\right\}$ ) such that $w^{\varepsilon}=w_{1}^{\varepsilon}+\varepsilon w_{2}^{\varepsilon}$, with

$$
\begin{aligned}
& \text { (i) } w_{1}^{\varepsilon} \rightarrow w \quad \text { weakly in } W^{1, p}(\Omega), \\
& \text { (ii) } \mathcal{T}_{\varepsilon}\left(w^{\varepsilon}\right) \rightarrow w \quad \text { weakly in } L^{p}\left(\Omega ; W^{1, p}(Y)\right), \\
& \text { (iii) } \mathcal{T}_{\varepsilon}\left(w_{2}^{\varepsilon}\right) \rightarrow \widehat{w} \quad \text { weakly in } L^{p}\left(\Omega ; W^{1, p}(Y)\right), \\
& \text { (iv) } \mathcal{T}_{\varepsilon}\left(\nabla w^{\varepsilon}\right) \rightarrow \nabla w+\nabla_{y} \widehat{w} \quad \text { weakly in } L^{p}(\Omega \times Y),
\end{aligned}
$$

where $\widehat{w}$ belongs to $L^{p}\left(\Omega ; W_{p e r}^{1, p}(Y)\right)$.

In section 5 we apply the periodic unfolding method to a classical periodic homogenization problem. We point out that, in the framework of this method, the proof of the homogenization result is elementary. It relies essentially on formula (1.1), on the properties of $\mathcal{T}_{\varepsilon}$, and on convergences (1.2). It applies directly for both homogeneous Dirichlet or Neumann boundary conditions without hypothesis on the regularity of $\partial \Omega$. For nonhomogenous boundary conditions (or for Robin-type condition), some regularity of $\partial \Omega$ is required for the problem to make sense, in which case the method applies also directly (see Remark 5.12).

Section 6 is devoted to a corrector result, which holds without any additional regularity on the data (contrary to all previous proofs; see [11], [30], and [59]). This result follows from the use of the averaging operator $\mathcal{U}_{\varepsilon}$. The idea of using averages to improve corrector results first appeared in Dal Maso and Defranceschi [33]. We also give some error estimates and a new corrector result for the case of domains with 
a smooth boundary (obtained by Griso in [42], [43], [44], and [45]). These results are explicitely connected to the unfolding method and improve on known classical ones (see [11] and [59]).

The periodic unfolding method is particularly well-suited for the case of multiscale problems. This is shown in section 7 by a simple backward iteration argument. This problem has a long history; one of the first papers on the subject is due to Bruggeman [19]. Its mathematical treatment by homogenization goes back to the book of Bensoussan, Lions, and Papanicolaou [11], where for this problem, the method of asymptotic expansions is used. For more recent references of multiscale homogenization and its applications, we refer to the books of Braides and Defranceschi [17], Milton [57], and the articles by Damlamian and Donato [35], Lukkassen and Milton [54], Lukkassen [53], Braides and Lukkassen [18], Babadjian and Baía [6], and Barchiesi [8].

The final section gives a list of papers where the method has been successfully applied since the publication of [24].

To conclude, let us turn back to the papers quoted at the beginning of this introduction and point out their relationships with our results. The dilation operation from Arbogast, Douglas, and Hornung [5] was defined in a domain which is an exact union of $\varepsilon Y$-cells. It consists in a change of variables, similar to that used in Definition 2.1 below. By this operation, any integral on $\Omega$ can be written as an integral over $\Omega \times Y$. Some elementary properties of the dilation operator in the space $L^{2}$ were also contained in Lemma 2 of [5].

The same dilation operator was used by Bourgeat, Luckhaus, and Mikelic in [16] under the name of "periodic modulation." Proposition 4.6 of [16] showed that if a sequence two-scale converges and its periodic modulation converges weakly, they have the same limit.

In the context of two-scale convergence, Allaire and Conca [3] defined a pair of extension and projection operators (suited to Bloch decompositions) which are adjoint of each other. They are similar to our operators $\mathcal{T}_{\varepsilon}$ and $\mathcal{U}_{\varepsilon}$ and the equivalent of property (2.12) and Proposition 2.18(ii) below, are proved in Lemma 4.2 of [3]. These properties were exploited by Allaire, Conca, and Vanninathan in [4] for a general bounded domain by extending all functions by zero on its complement.

In [48], Lenczner used the dilation operator (here called "two-scale transformation") in order to treat the homogenization of discrete electrical networks (by nature, the domain is a union of $\varepsilon$-cells). The convergence of the two-scale transform is called two-scale convergence (this would be confusing except that it was shown to be equivalent to the original two-scale convergence). As an aside, a convergence similar to (1.2)(iv) was also treated. In Lenczner and Mercier [49], Lenczner and SenouciBereksi [50], and Lenczner, Kader, and Perrier [51], this theory was applied to periodic electrical networks.

Finally, Casado Díaz and Luna-Laynez [21], Casado Díaz, Luna-Laynez, and Martin [22] and [23] used the dilation operator in the case of reticulated structures. In this framework, they obtained the equivalent of (3.7)(i) of Theorem 3.5 below.

\section{Unfolding in $L^{p}$-spaces.}

2.1. The unfolding operator $\mathcal{T}_{\varepsilon}$. In $\mathbb{R}^{n}$, let $\Omega$ be an open set and $Y$ a reference cell (e.g., $] 0,1[n$, or more generally, a set having the paving property, with respect to a basis $\left(b_{1}, \ldots, b_{n}\right)$ defining the periods).

By analogy with the notation in the one-dimensional case, for $z \in \mathbb{R}^{n},[z]_{Y}$ denotes the unique integer combination $\sum_{j=1}^{n} k_{j} b_{j}$ of the periods such that $z-[z]_{Y}$ belongs 


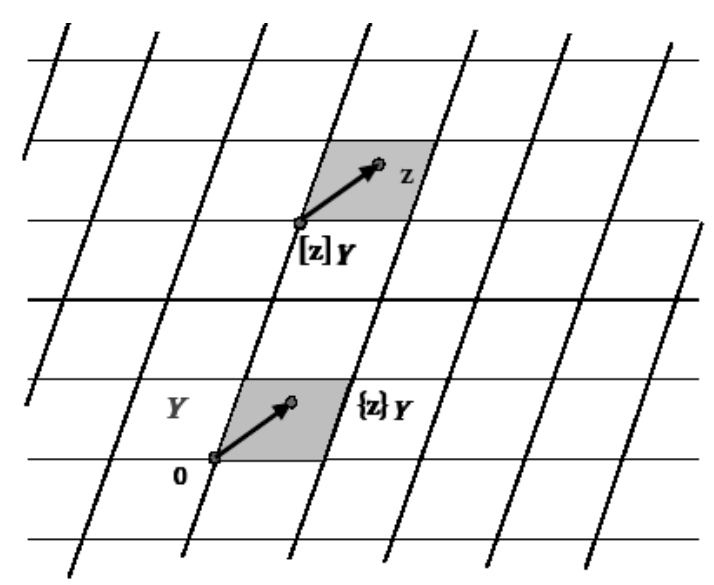

FIG. 1. Definition of $[z]_{Y}$ and $\{z\}_{Y}$.

to $Y$, and set

$$
\{z\}_{Y}=z-[z]_{Y} \in Y \quad \text { a.e. for } \quad z \in \mathbb{R}^{n} .
$$

Then for each $x \in \mathbb{R}^{n}$, one has

$$
x=\varepsilon\left(\left[\frac{x}{\varepsilon}\right]_{Y}+\left\{\frac{x}{\varepsilon}\right\}_{Y}\right) \text { a.e. for } \quad x \in \mathbb{R}^{n} \text { (See Figure 1). }
$$

We use the following notations:

$$
\left\{\begin{array}{l}
\Xi_{\varepsilon}=\left\{\xi \in \mathbb{Z}^{N}, \varepsilon(\xi+Y) \subset \Omega\right\}, \\
\widehat{\Omega}_{\varepsilon}=\text { interior }\left\{\bigcup_{\xi \in \Xi_{\varepsilon}} \varepsilon(\xi+\bar{Y})\right\}, \\
\Lambda_{\varepsilon}=\Omega \backslash \widehat{\Omega}_{\varepsilon} .
\end{array}\right.
$$

The set $\widehat{\Omega}_{\varepsilon}$ is the largest union of $\varepsilon(\xi+\bar{Y})$ cells $\left(\xi \in \mathbb{Z}^{n}\right)$ included in $\Omega$, while $\Lambda_{\varepsilon}$ is the subset of $\Omega$ containing the parts from $\varepsilon(\xi+\bar{Y})$ cells intersecting the boundary $\partial \Omega$ (see Figure 2).

DEFINITION 2.1. For $\phi$ Lebesgue-measurable on $\Omega$, the unfolding operator $\mathcal{T}_{\varepsilon}$ is defined as follows:

$$
\mathcal{T}_{\varepsilon}(\phi)(x, y)= \begin{cases}\phi\left(\varepsilon\left[\frac{x}{\varepsilon}\right]_{Y}+\varepsilon y\right) & \text { a.e. for }(x, y) \in \widehat{\Omega}_{\varepsilon} \times Y, \\ 0 & \text { a.e. for }(x, y) \in \Lambda_{\varepsilon} \times Y .\end{cases}
$$

Observe that the function $\mathcal{T}_{\varepsilon}(\phi)$ is Lebesgue-measurable on $\Omega \times Y$ and vanishes for $x$ outside of the set $\widehat{\Omega}_{\varepsilon}$.

As in classical periodic homogenization, two different scales appear in the definition of $\mathcal{T}_{\varepsilon}$ : the "macroscopic" scale $x$ gives the position of a point in the domain $\Omega$, while the "microscopic" scale $y(=x / \varepsilon)$ gives the position of a point in the cell $Y$. The unfolding operator doubles the dimension of the space and puts all of the oscillations in the second variable, in this way separating the two scales (see Figures 3, 4 and Figures 5,6).

The following property of $\mathcal{T}_{\varepsilon}$ is a simple consequence of Definition 2.1 for $v$ and $w$ Lebesgue-measurable; it will be used extensively:

$$
\mathcal{T}_{\varepsilon}(v w)=\mathcal{T}_{\varepsilon}(v) \mathcal{T}_{\varepsilon}(w)
$$




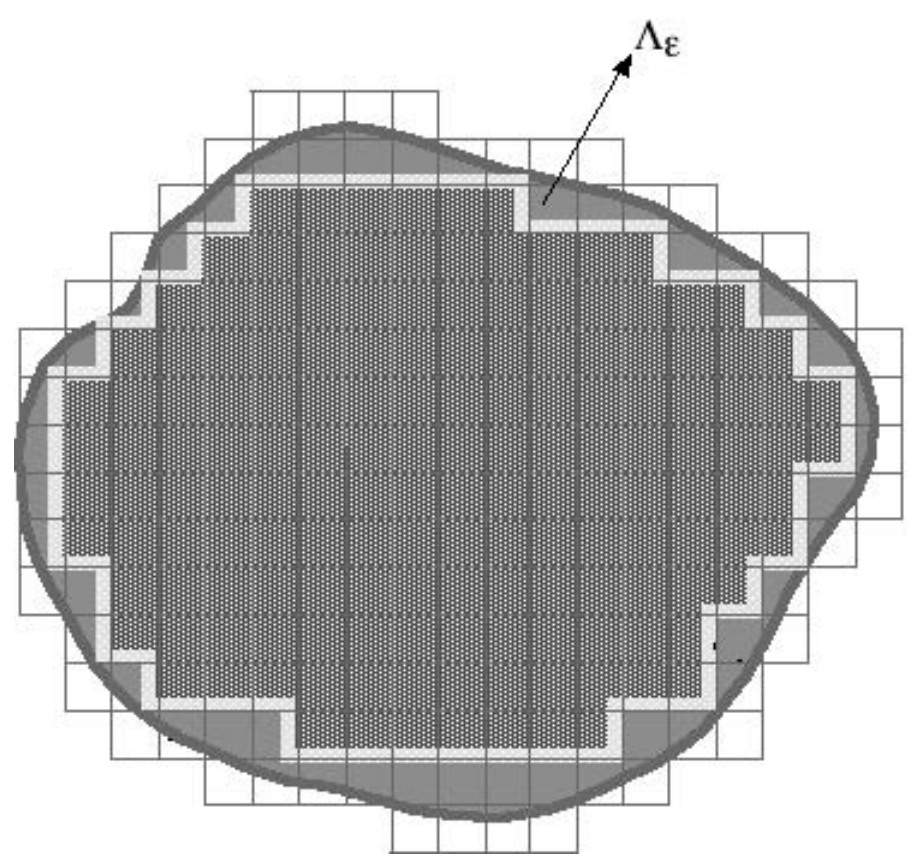

Fig. 2. The domains $\widehat{\Omega}_{\varepsilon}$ and $\Lambda_{\varepsilon}$.

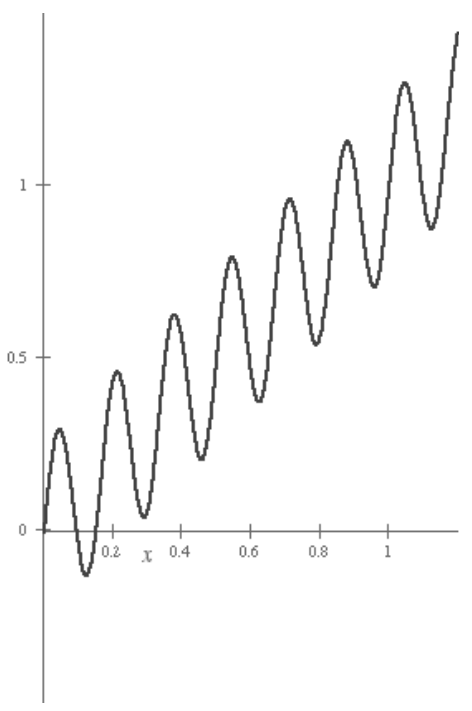

FIG. 3. $f_{\varepsilon}(x)=\frac{1}{4} \sin \left(2 \pi \frac{x}{\varepsilon}\right)+x ; \varepsilon=\frac{1}{6}$.

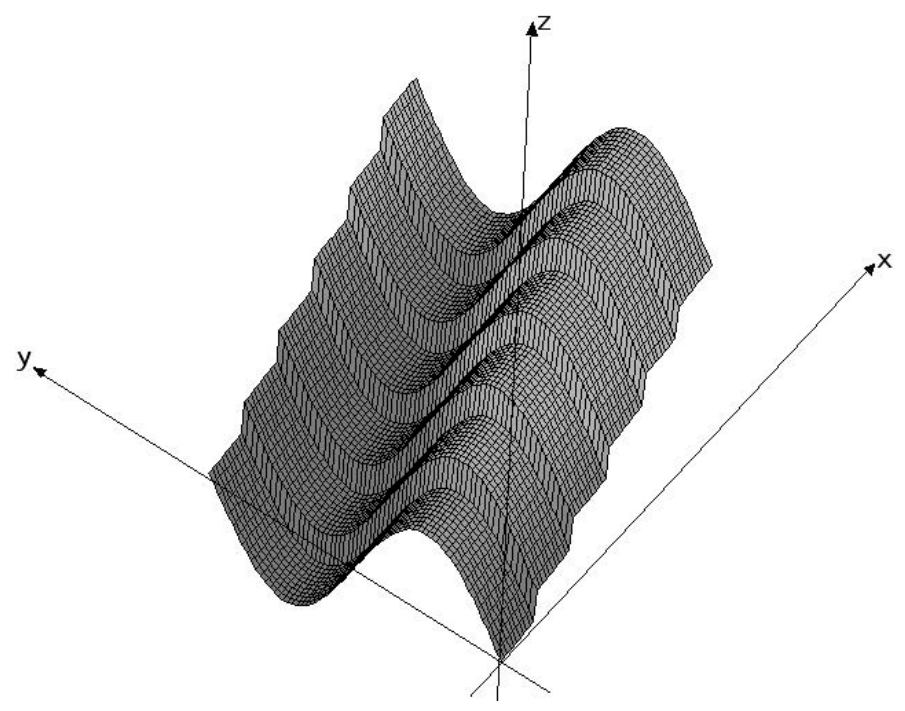

FIG. 4. $\mathcal{T}_{\varepsilon}\left(f_{\varepsilon}\right)$. 


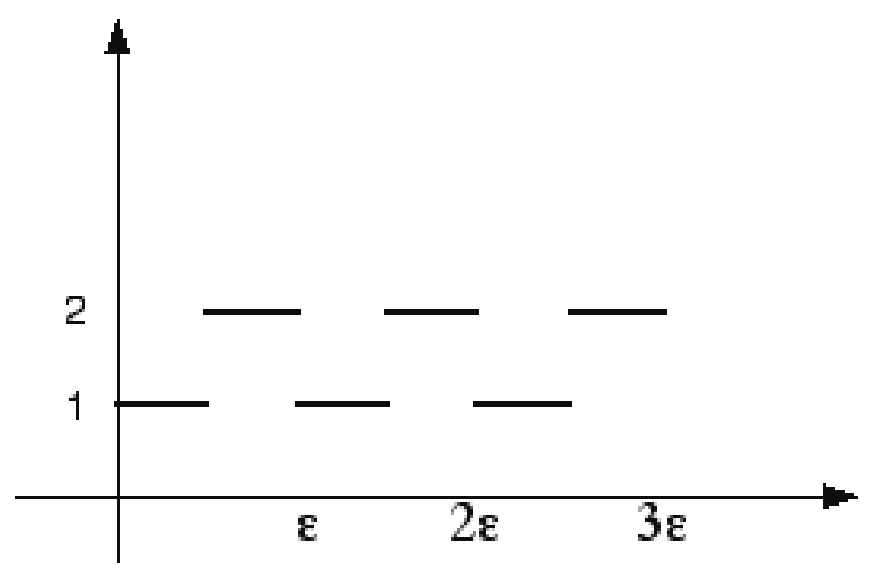

FIG. 5. $f_{\varepsilon}=f\left(\left\{\frac{x}{\varepsilon}\right\}_{Y}\right)$.

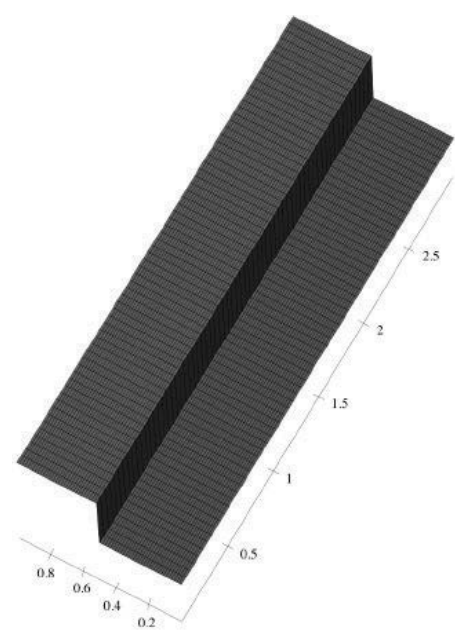

FIG. 6. $\mathcal{T}_{\varepsilon}\left(f_{\varepsilon}\right)$.

Another simple consequence of Definition 2.1 is the following result concerning highly oscillating functions.

Proposition 2.2. For $f$ measurable on $Y$, extended by $Y$-periodicity to the whole of $\mathbb{R}^{n}$, define the sequence $\left\{f_{\varepsilon}\right\}$ by

$$
f_{\varepsilon}(x)=f\left(\frac{x}{\varepsilon}\right) \quad \text { a.e. for } x \in \mathbb{R}^{n} .
$$

Then

$$
\mathcal{T}_{\varepsilon}\left(\left.f_{\varepsilon}\right|_{\Omega}\right)(x, y)=\left\{\begin{array}{lll}
f(y) & \text { a.e. for } & (x, y) \in \widehat{\Omega}_{\varepsilon} \times Y \\
0 & \text { a.e. for } & (x, y) \in \Lambda_{\varepsilon} \times Y .
\end{array}\right.
$$

If $f$ belongs to $L^{p}(Y), p \in[1,+\infty[$, and if $\Omega$ is bounded,

$$
\mathcal{T}_{\varepsilon}\left(\left.f_{\varepsilon}\right|_{\Omega}\right) \rightarrow f \quad \text { strongly in } L^{p}(\Omega \times Y) .
$$

Remark 2.3. An equivalent way to define $f_{\varepsilon}$ in $(2.3)$ is to take simply $f_{\varepsilon}(x)=$ $f\left(\left\{\frac{x}{\varepsilon}\right\}_{Y}\right)$. For example, with

$$
f(y)= \begin{cases}1 & \text { for } y \in(0,1 / 2) \\ 2 & \text { for } y \in(1 / 2,1)\end{cases}
$$

$f_{\varepsilon}$ is the highly oscillating periodic function, with period $\varepsilon$ from Figure 5 . 
Remark 2.4. Let $f$ in $L^{p}(Y), p \in\left[1,+\infty\left[\right.\right.$, and $f_{\varepsilon}$ be defined by (2.3). It is well-known that $\left\{\left.f_{\varepsilon}\right|_{\Omega}\right\}$ converges weakly in $L^{p}(\Omega)$ to the mean value of $f$ on $Y$, and not strongly unless $f$ is a constant (see Remark 2.11 below).

The next two results, essential in the study of the properties of the unfolding operator, are also straightforward from Definition 2.1.

Proposition 2.5. For $p \in\left[1,+\infty\left[\right.\right.$, the operator $\mathcal{T}_{\varepsilon}$ is linear and continuous from $L^{p}(\Omega)$ to $L^{p}(\Omega \times Y)$. For every $\phi$ in $L^{1}(\Omega)$ and $w$ in $L^{p}(\Omega)$,

(i) $\frac{1}{|Y|} \int_{\Omega \times Y} \mathcal{T}_{\varepsilon}(\phi)(x, y) d x d y=\int_{\Omega} \phi(x) d x-\int_{\Lambda_{\varepsilon}} \phi(x) d x=\int_{\widehat{\Omega}_{\varepsilon}} \phi(x) d x$,

(ii) $\frac{1}{|Y|} \int_{\Omega \times Y}\left|\mathcal{T}_{\varepsilon}(\phi)\right| d x d y \leq \int_{\Omega}|\phi| d x$,

(iii) $\left|\int_{\Omega} \phi d x-\frac{1}{|Y|} \int_{\Omega \times Y} \mathcal{T}_{\varepsilon}(\phi) d x d y\right| \leq \int_{\Lambda_{\varepsilon}}|\phi| d x$,

(iv) $\left\|\mathcal{T}_{\varepsilon}(w)\right\|_{L^{p}(\Omega \times Y)}=|Y|^{\frac{1}{p}}\left\|w 1_{\widehat{\Omega}_{\varepsilon}}\right\|_{L^{p}(\Omega)} \leq|Y|^{\frac{1}{p}}\|w\|_{L^{p}(\Omega)}$.

Proof. Recalling Definition 2.2 of $\widehat{\Omega}_{\varepsilon}$, one has

$$
\begin{aligned}
\frac{1}{|Y|} \int_{\Omega \times Y} \mathcal{T}_{\varepsilon}(\phi)(x, y) d x d y & =\frac{1}{|Y|} \int_{\widehat{\Omega}_{\varepsilon} \times Y} \mathcal{T}_{\varepsilon}(\phi)(x, y) d x d y \\
& =\frac{1}{|Y|} \sum_{\xi \in \Xi_{\varepsilon}} \int_{(\varepsilon \xi+\varepsilon Y) \times Y} \mathcal{T}_{\varepsilon}(\phi)(x, y) d x d y .
\end{aligned}
$$

On each $(\varepsilon \xi+\varepsilon Y) \times Y$, by definition, $\mathcal{T}_{\varepsilon}(\phi)(x, y)=\phi(\varepsilon \xi+\varepsilon y)$ is constant in $x$. Hence, each integral in the sum on the right-hand side successively equals

$$
\begin{aligned}
\int_{(\varepsilon \xi+\varepsilon Y) \times Y} \mathcal{T}_{\varepsilon}(\phi)(x, y) d x d y & =|\varepsilon \xi+\varepsilon Y| \int_{Y} \phi(\varepsilon \xi+\varepsilon y) d y \\
& =\varepsilon^{n}|Y| \int_{Y} \phi(\varepsilon \xi+\varepsilon y) d y=|Y| \int_{(\varepsilon \xi+\varepsilon Y)} \phi(x) d x .
\end{aligned}
$$

By summing over $\Xi_{\varepsilon}$, the right-hand side becomes $\int_{\widehat{\Omega}_{\varepsilon}} \phi(x) d x$, which gives the result.

Property (iii) in Proposition 2.5 shows that any integral of a function on $\Omega$ is "almost equivalent" to the integral of its unfolded on $\Omega \times Y$; the "integration defect" arises only from the cells intersecting the boundary $\partial \Omega$ and is controlled by its integral over $\Lambda_{\varepsilon}$.

The next proposition, which we call unfolding criterion for integrals (u.c.i.), is a very useful tool when treating homogenization problems.

Proposition 2.6 (u.c.i.). If $\left\{\phi_{\varepsilon}\right\}$ is a sequence in $L^{1}(\Omega)$ satisfying

$$
\int_{\Lambda_{\varepsilon}}\left|\phi_{\varepsilon}\right| d x \rightarrow 0
$$

then

$$
\int_{\Omega} \phi_{\varepsilon} d x-\frac{1}{|Y|} \int_{\Omega \times Y} \mathcal{T}_{\varepsilon}\left(\phi_{\varepsilon}\right) d x d y \rightarrow 0 .
$$

Based on this result, we introduce the following notation. 
Notation. If $\left\{w_{\varepsilon}\right\}$ is a sequence satisfying u.c.i., we write

$$
\int_{\Omega} w_{\varepsilon} d x \stackrel{\mathcal{T}_{\varepsilon}}{\simeq} \frac{1}{|Y|} \int_{\Omega \times Y} \mathcal{T}_{\varepsilon}\left(w_{\varepsilon}\right) d x d y
$$

Proposition 2.7. Let $\left\{u_{\varepsilon}\right\}$ be a bounded sequence in $L^{p}(\Omega)$, with $\left.\left.p \in\right] 1,+\infty\right]$ and $v \in L^{p^{\prime}}(\Omega)\left(1 / p+1 / p^{\prime}=1\right)$, then

$$
\int_{\Omega} u_{\varepsilon} v d x \stackrel{\mathcal{T}_{\varepsilon}}{\simeq} \frac{1}{|Y|} \int_{\Omega \times Y} \mathcal{T}_{\varepsilon}\left(u_{\varepsilon}\right) \mathcal{T}_{\varepsilon}(v) d x d y
$$

Suppose $\partial \Omega$ is bounded. Let $\left\{u_{\varepsilon}\right\}$ be a bounded sequence in $L^{p}(\Omega)$ and $\left\{v_{\varepsilon}\right\}$ a bounded sequence in $L^{q}(\Omega)$, with $1 / p+1 / q<1$, then

$$
\int_{\Omega} u_{\varepsilon} v_{\varepsilon} d x \stackrel{\mathcal{I}_{\varepsilon}}{\simeq} \frac{1}{|Y|} \int_{\Omega \times Y} \mathcal{T}_{\varepsilon}\left(u_{\varepsilon}\right) \mathcal{T}_{\varepsilon}\left(v_{\varepsilon}\right) d x d y .
$$

Proof. Observe that $1_{\Lambda_{\varepsilon}}(x) \rightarrow 0$ for all $x \in \Omega$. Consequently, by the Lebesgue dominated convergence theorem, one gets $\int_{\Lambda_{\varepsilon}}|v|^{p^{\prime}} d x \rightarrow 0$, and then by the Hölder inequality, $\int_{\Lambda_{\varepsilon}}\left|u_{\varepsilon} v\right| d x \rightarrow 0$. This proves (2.5). If $\partial \Omega$ is bounded, then one immediately has $\left|\Lambda_{\varepsilon}\right| \rightarrow 0$ when $\varepsilon \rightarrow 0$, and this implies (2.6).

We now investigate the convergence properties related to the unfolding operator when $\varepsilon \rightarrow 0$. For $\phi$ uniformly continuous on $\Omega$, with modulus of continuity $m_{\phi}$, it is easy to see that

$$
\sup _{x \in \widehat{\Omega}_{\varepsilon}, y \in Y}\left|\mathcal{T}_{\varepsilon}(\phi)(x, y)-\phi(x)\right| \leq m_{\phi}(\varepsilon)
$$

So, as $\varepsilon$ goes to zero, even though $\mathcal{T}_{\varepsilon}(\phi)$ is not continuous, it converges to $\phi$ uniformly on any open set strongly included in $\Omega$. By density, and making use of Proposition 2.5, further convergence properties can be expressed using the mean value of a function defined on $\Omega \times Y$.

Definition 2.8. The mean value operator $\mathcal{M}_{Y}: L^{p}(\Omega \times Y) \mapsto L^{p}(\Omega)$ for $p \in$ $[1,+\infty]$, is defined as follows:

$$
\mathcal{M}_{Y}(\Phi)(x)=\frac{1}{|Y|} \int_{Y} \Phi(x, y) d y \quad \text { a.e. for } \quad x \in \Omega
$$

Observe that an immediate consequence of this definition is the estimate

$$
\left\|\mathcal{M}_{Y}(\Phi)\right\|_{L^{p}(\Omega)} \leq|Y|^{-\frac{1}{p}}\|\Phi\|_{L^{p}(\Omega \times Y)} \quad \text { for every } \Phi \in L^{p}(\Omega \times Y) .
$$

Proposition 2.9. Let $p$ belong to $[1,+\infty[$.

(i) For $w \in L^{p}(\Omega)$,

$$
\mathcal{T}_{\varepsilon}(w) \rightarrow w \quad \text { strongly in } L^{p}(\Omega \times Y)
$$

(ii) Let $\left\{w_{\varepsilon}\right\}$ be a sequence in $L^{p}(\Omega)$ such that

$$
w_{\varepsilon} \rightarrow w \quad \text { strongly in } L^{p}(\Omega) .
$$

Then

$$
\mathcal{T}_{\varepsilon}\left(w_{\varepsilon}\right) \rightarrow w \quad \text { strongly in } L^{p}(\Omega \times Y) \text {. }
$$


(iii) For every relatively weakly compact sequence $\left\{w_{\varepsilon}\right\}$ in $L^{p}(\Omega)$, the corresponding $\left\{\mathcal{T}_{\varepsilon}\left(w_{\varepsilon}\right)\right\}$ is relatively weakly compact in $L^{p}(\Omega \times Y)$. Furthermore, if

$$
\mathcal{T}_{\varepsilon}\left(w_{\varepsilon}\right) \rightarrow \widehat{w} \quad \text { weakly in } L^{p}(\Omega \times Y),
$$

then

$$
w_{\varepsilon} \rightarrow \mathcal{M}_{Y}(\widehat{w}) \quad \text { weakly in } L^{p}(\Omega) .
$$

(iv) If $\mathcal{T}_{\varepsilon}\left(w_{\varepsilon}\right) \rightarrow \widehat{w}$ weakly in $L^{p}(\Omega \times Y)$, then

$$
\|\widehat{w}\|_{L^{p}(\Omega \times Y)} \leq \liminf _{\varepsilon \rightarrow 0}|Y|^{\frac{1}{p}}\left\|w_{\varepsilon}\right\|_{L^{p}(\Omega)} .
$$

(v) Suppose $p>1$, and let $\left\{w_{\varepsilon}\right\}$ be a bounded sequence in $L^{p}(\Omega)$. Then, the following assertions are equivalent:

(a) $\mathcal{T}_{\varepsilon}\left(w_{\varepsilon}\right) \rightarrow \widehat{w} \quad$ weakly in $L^{p}(\Omega \times Y) \quad$ and $\limsup _{\varepsilon \rightarrow 0}|Y|^{\frac{1}{p}}\left\|w_{\varepsilon}\right\|_{L^{p}(\Omega)} \leq$ $\|\widehat{w}\|_{L^{p}(\Omega \times Y)}$,

(b) $\mathcal{T}_{\varepsilon}\left(w_{\varepsilon}\right) \rightarrow \widehat{w} \quad$ strongly in $L^{p}(\Omega \times Y) \quad$ and $\int_{\Lambda_{\varepsilon}}\left|w_{\varepsilon}\right|^{p} d x \rightarrow 0$.

Proof. (i) The result is obvious for any $w \in \mathcal{D}(\Omega)$. If $w \in L^{p}(\Omega)$, let $\phi \in \mathcal{D}(\Omega)$. Then, by using (iv) from Proposition 2.5,

$$
\begin{aligned}
\left\|\mathcal{T}_{\varepsilon}(w)-w\right\|_{L^{p}(\Omega \times Y)} & =\left\|\mathcal{T}_{\varepsilon}(w-\phi)+\left(\mathcal{T}_{\varepsilon}(\phi)-\phi\right)+(\phi-w)\right\|_{L^{p}(\Omega \times Y)} \\
& \leq 2|Y|^{\frac{1}{p}}\|w-\phi\|_{L^{p}(\Omega)}+\left\|\mathcal{T}_{\varepsilon}(\phi)-\phi\right\|_{L^{p}(\Omega \times Y)},
\end{aligned}
$$

hence,

$$
\limsup _{\varepsilon \rightarrow 0}\left\|\mathcal{T}_{\varepsilon}(w)-w\right\|_{L^{p}(\Omega \times Y)} \leq 2|Y|^{\frac{1}{p}}\|w-\phi\|_{L^{p}(\Omega)},
$$

from which statement (i) follows by density.

(ii) The following estimate, a consequence of Proposition 2.5(iv), gives the result

$$
\left\|\mathcal{T}_{\varepsilon}\left(w_{\varepsilon}\right)-\mathcal{T}_{\varepsilon}(w)\right\|_{L^{p}(\Omega \times Y)} \leq|Y|^{\frac{1}{p}}\left\|w_{\varepsilon}-w\right\|_{L^{p}(\Omega)} \quad \forall w \in L^{p}(\Omega) .
$$

(iii) For $p \in] 1,+\infty\left[\right.$, by Proposition 2.5(iv), boundedness is preserved by $\mathcal{T}_{\varepsilon}$. Suppose that $\mathcal{T}_{\varepsilon}\left(w_{\varepsilon}\right) \rightarrow \widehat{w}$ weakly in $L^{p}(\Omega \times Y)$, and let $\psi \in L^{p^{\prime}}(\Omega)$. From Proposition 2.7,

$$
\int_{\Omega} w_{\varepsilon}(x) \psi(x) d x \stackrel{\mathcal{I}_{\varepsilon}}{\simeq} \frac{1}{|Y|} \int_{\Omega \times Y} \mathcal{T}_{\varepsilon}\left(w_{\varepsilon}\right)(x, y) \mathcal{T}_{\varepsilon}(\psi)(x, y) d x d y .
$$

In view of (i), one can pass to the limit in the right-hand side to obtain

$$
\lim _{\varepsilon \rightarrow 0} \int_{\Omega} w_{\varepsilon}(x) \psi(x) d x=\int_{\Omega}\left\{\frac{1}{|Y|} \int_{Y} \widehat{w}(x, y) d y\right\} \psi(x) d x .
$$

For $p=1$, one uses the extra property satisfied by weakly convergent sequences in $L^{1}(\Omega)$, in the form of the De La Vallée-Poussin criterion (which is equivalent to 
relative weak compactness): there exists a continuous convex function $\Phi: \mathbb{R}^{+} \mapsto \mathbb{R}^{+}$ such that

$$
\lim _{t \rightarrow+\infty} \frac{\Phi(t)}{t}=+\infty, \quad \text { and the set }\left\{\int_{\Omega}\left(\Phi \circ\left|w_{\varepsilon}\right|\right)(x) d x\right\} \text { is bounded. }
$$

Unfolding the last integral shows that

$$
\left\{\int_{\Omega \times Y}\left(\Phi \circ\left|\mathcal{T}_{\varepsilon}\left(w_{\varepsilon}\right)\right|\right)(x, y) d x d y\right\} \text { is bounded, }
$$

which completes the proof of weak compactness of $\left\{\mathcal{T}_{\varepsilon}\left(w_{\varepsilon}\right)\right\}$ in $L^{1}(\Omega \times Y)$ in the case of $\Omega$ with finite measure. For the case where the measure of $\Omega$ is not finite, a similar argument shows that the equiintegrability at infinity of the sequence $\left\{w_{\varepsilon}\right\}$ carries over to $\left\{\mathcal{T}_{\varepsilon}\left(w_{\varepsilon}\right)\right\}$.

If $\mathcal{T}_{\varepsilon}\left(w_{\varepsilon}\right) \rightarrow \widehat{w}$ weakly in $L^{1}(\Omega \times Y)$, let $\psi$ be in $\mathcal{D}(\Omega)$. For $\varepsilon$ sufficiently small, one has

$$
\int_{\Omega} w_{\varepsilon}(x) \psi(x) d x=\frac{1}{|Y|} \int_{\Omega \times Y} \mathcal{T}_{\varepsilon}\left(w_{\varepsilon}\right)(x, y) \mathcal{T}_{\varepsilon}(\psi)(x, y) d x d y
$$

In view of (i), one can pass to the limit in the right-hand side to obtain

$$
\lim _{\varepsilon \rightarrow 0} \int_{\Omega} w_{\varepsilon}(x) \psi(x) d x=\int_{\Omega}\left\{\frac{1}{|Y|} \int_{Y} \widehat{w}(x, y) d y\right\} \psi(x) d x .
$$

(iv) Inequality (2.8) is a simple consequence of Proposition 2.5(ii).

(v) Proposition 2.5(i) applied to the function $\left|w_{\varepsilon}\right|^{p}$ gives

$$
\frac{1}{|Y|}\left\|\mathcal{T}_{\varepsilon}\left(w_{\varepsilon}\right)\right\|_{L^{p}(\Omega \times Y)}^{p}+\int_{\Lambda_{\varepsilon}}\left|w_{\varepsilon}\right|^{p} d x=\left\|w_{\varepsilon}\right\|_{L^{p}(\Omega)}^{p} .
$$

This identity implies the required equivalence.

Corollary 2.10. Let $f$ be in $L^{p}(Y), p \in\left[1,+\infty\left[\right.\right.$, and $\left\{f_{\varepsilon}\right\}$ be the sequence defined by (2.3). Then

$$
\left.f_{\varepsilon}\right|_{\Omega} \rightarrow \mathcal{M}_{Y}(f) \quad \text { weakly in } L^{p}(\Omega)
$$

Proof. Proposition 2.2 gives the strong (hence weak) convergenge of $\left\{\mathcal{T}_{\varepsilon}\left(f_{\varepsilon} \mid \Omega\right)\right\}$ to $f$ in $L^{p}(\Omega \times Y)$. Convergence (2.9) follows from Proposition 2.9(iii). ${ }^{1}$

Remark 2.11. In general, in the case where $\Lambda_{\varepsilon}$ is not null set (for every $\varepsilon$ ), the strong (resp. weak) convergence of the sequence $\left\{\mathcal{T}_{\varepsilon}\left(w_{\varepsilon}\right)\right\}$ does not imply the corresponding convergence for the sequence $\left\{w_{\varepsilon}\right\}$, since it gives no control of the sequence $\left\{w_{\varepsilon} 1_{\Lambda_{\varepsilon}}\right\}$. If $\left\{w_{\varepsilon} 1_{\Lambda_{\varepsilon}}\right\}$ is bounded in $L^{p}(\Omega)$ and if $\left\{\mathcal{T}_{\varepsilon}\left(w_{\varepsilon}\right)\right\}$ converges weakly, so does $\left\{w_{\varepsilon}\right\}$ by Proposition 2.9(iii). On the other hand, even if $\left\{w_{\varepsilon} 1_{\Lambda_{\varepsilon}}\right\}$ converges strongly to 0 in $L^{p}(\Omega)$, the strong convergence of $\left\{\mathcal{T}_{\varepsilon}\left(w_{\varepsilon}\right)\right\}$ does not imply that of $\left\{w_{\varepsilon}\right\}$ as it is shown by the sequence $\left\{\left.f_{\varepsilon}\right|_{\Omega}\right\}$ in Corollary 2.10, unless $f$ is a constant on $Y$.

Corollary 2.12. Let pelong to $] 1,+\infty\left[\right.$, let $\left\{u_{\varepsilon}\right\}$ be a sequence in $L^{p}(\Omega)$ such that

$$
\mathcal{T}_{\varepsilon}\left(u_{\varepsilon}\right) \rightarrow u \quad \text { weakly in } L^{p}(\Omega \times Y)
$$

\footnotetext{
${ }^{1}$ Note that the proof of convergence (2.9) is really straightforward when using unfolding!
} 
and let $\left\{v_{\varepsilon}\right\}$ be a sequence in $L^{p^{\prime}}(\Omega)\left(1 / p+1 / p^{\prime}=1\right)$, with

$$
\mathcal{T}_{\varepsilon}\left(v_{\varepsilon}\right) \rightarrow v \quad \text { strongly in } L^{p^{\prime}}(\Omega \times Y) .
$$

Then, for any $\varphi$ in $C_{c}(\Omega)$, one has

$$
\int_{\Omega} u_{\varepsilon}(x) v_{\varepsilon}(x) \varphi(x) d x \rightarrow \frac{1}{|Y|} \int_{\Omega \times Y} u(x, y) v(x, y) \varphi(x) d x d y .
$$

Moreover, if

$$
\int_{\Lambda_{\varepsilon}}\left|v_{\varepsilon}\right|^{p^{\prime}} d x \rightarrow 0
$$

then, for any $\varphi$ in $C(\bar{\Omega})$, one has

$$
\int_{\Omega} u_{\varepsilon}(x) v_{\varepsilon}(x) \varphi(x) d x \rightarrow \frac{1}{|Y|} \int_{\Omega \times Y} u(x, y) v(x, y) \varphi(x) d x d y .
$$

Proof. The result follows from the fact that, in both cases, the sequence $\left\{u_{\varepsilon} v_{\varepsilon} \phi\right\}$ satisfies the u.c.i. by the Hölder inequality.

Remark 2.13. A consequence of (iii) of Proposition 2.9, together with (iv) of Proposition 2.5, is the following. Suppose the sequence $\left\{w_{\varepsilon}\right\}$ converges weakly to $w$ in $L^{p}(\Omega)$. Then the sequence $\left\{\mathcal{T}_{\varepsilon}\left(w_{\varepsilon}\right)\right\}$ is relatively weakly compact in $L^{p}(\Omega \times Y)$, and each of its weak-limit points $\widehat{w}$ satisfies $\mathcal{M}_{Y}(\widehat{w})=w$.

Now recall the following definition from Nguetseng [58] and Allaire [1].

Two-scale convergence. Let $p \in] 1,+\infty\left[\right.$. A bounded sequence $\left\{w_{\varepsilon}\right\}$ in $L^{p}(\Omega)$ two-scale converges to some $w$ belonging to $L^{p}(\Omega \times Y)$, whenever, for every smooth function $\varphi$ on $\Omega \times Y$, the following convergence holds:

$$
\int_{\Omega} w_{\varepsilon}(x) \varphi\left(x, \frac{x}{\varepsilon}\right) d x \rightarrow \frac{1}{|Y|} \iint_{\Omega \times Y} w(x, y) \varphi(x, y) d x d y .
$$

The next result reduces two-scale convergence of a sequence to a mere weak $L^{p}(\Omega \times Y)$-convergence of the unfolded sequence.

Proposition 2.14. Let $\left\{w_{\varepsilon}\right\}$ be a bounded sequence in $L^{p}(\Omega)$, with $\left.p \in\right] 1,+\infty[$. The following assertions are equivalent:

(i) $\left\{\mathcal{T}_{\varepsilon}\left(w_{\varepsilon}\right)\right\}$ converges weakly to $w$ in $L^{p}(\Omega \times Y)$,

(ii) $\left\{w_{\varepsilon}\right\}$ two-scale converges to $w$.

Proof. To prove this equivalence, it is enough to check that, for every $\varphi$ in a set of admissible test functions for two-scale convergence (for instance, $\mathcal{D}\left(\Omega, L^{q}(Y)\right)$ ), the sequence $\left\{\mathcal{T}_{\varepsilon}[\varphi(x, x / \varepsilon)]\right\}$ converges strongly to $\varphi$ in $\left.L^{q}(\Omega \times Y)\right)$. This follows from the definition of $\mathcal{T}_{\varepsilon}$, indeed

$$
\mathcal{T}_{\varepsilon}\left[\varphi\left(x, \frac{x}{\varepsilon}\right)\right](x, y)=\varphi\left(\varepsilon\left[\frac{x}{\varepsilon}\right]_{Y}+\varepsilon y, y\right) .
$$

Remark 2.15. Proposition 2.14 shows that the two-scale convergence of a sequence in $\left.L^{p}(\Omega), p \in\right] 1,+\infty\left[\right.$, is equivalent to the weak $-L^{p}(\Omega \times Y)$ convergence of the unfolded sequence. Notice that, by definition, to check the two-scale convergence, one has to use special test functions. To check a weak convergence in the space $L^{p}(\Omega \times Y)$, one simply makes the use of functions in the dual space $L^{p^{\prime}}(\Omega \times Y)$. Moreover, due to density properties, it is sufficient to check this convergence only on smooth functions from $\mathcal{D}(\Omega \times Y)$. 
2.2. The averaging operator $\mathcal{U}_{\varepsilon}$. In this section, we consider the adjoint $\mathcal{U}_{\varepsilon}$ of $\mathcal{T}_{\varepsilon}$, which we call averaging operator. In order to do so, let $v$ be in $L^{p}(\Omega \times Y)$, and let $u$ be in $L^{p^{\prime}}(\Omega)$. We have successively,

$$
\begin{aligned}
\frac{1}{|Y|} \int_{\Omega \times Y} \mathcal{T}_{\varepsilon}(u)(x, y) & v(x, y) d x d y=\frac{1}{|Y|} \int_{\widehat{\Omega}_{\varepsilon} \times Y} \mathcal{T}_{\varepsilon}(u)(x, y) v(x, y) d x d y \\
= & \frac{1}{|Y|} \sum_{\xi \in \Xi_{\varepsilon}} \int_{\varepsilon(\xi+Y) \times Y} u(\varepsilon \xi+\varepsilon y) v(x, y) d x d y \\
& =\sum_{\xi \in \Xi_{\varepsilon}} \frac{1}{|Y|} \int_{Y \times Y} u(\varepsilon \xi+\varepsilon y) v(\varepsilon \xi+\varepsilon z, y) \varepsilon^{N} d z d y \\
& =\sum_{\xi \in \Xi_{\varepsilon}} \frac{1}{|Y|} \int_{Y} d z \int_{\varepsilon(\xi+Y)} u(x) v\left(\varepsilon\left[\frac{x}{\varepsilon}\right]_{Y}+\varepsilon z,\left\{\frac{x}{\varepsilon}\right\}_{Y}\right) d x \\
& =\int_{\widehat{\Omega}_{\varepsilon}} u(x)\left(\frac{1}{|Y|} \int_{Y} v\left(\varepsilon\left[\frac{x}{\varepsilon}\right]_{Y}+\varepsilon z,\left\{\frac{x}{\varepsilon}\right\}_{Y}\right) d z\right) d x .
\end{aligned}
$$

This gives the formula for the averaging operator $\mathcal{U}_{\varepsilon}$.

DeFinition 2.16. For $p$ in $[1,+\infty]$, the averaging operator $\mathcal{U}_{\varepsilon}: L^{p}(\Omega \times Y) \mapsto$ $L^{p}(\Omega)$ is defined as

$$
\mathcal{U}_{\varepsilon}(\Phi)(x)= \begin{cases}\frac{1}{|Y|} \int_{Y} \Phi\left(\varepsilon\left[\frac{x}{\varepsilon}\right]_{Y}+\varepsilon z,\left\{\frac{x}{\varepsilon}\right\}_{Y}\right) d z & \text { a.e. for } x \in \widehat{\Omega}_{\varepsilon} \\ 0 & \text { a.e. for } x \in \Lambda_{\varepsilon} .\end{cases}
$$

Consequently, for $\psi \in L^{p}(\Omega)$ and $\Phi \in L^{p^{\prime}}(\Omega \times Y)$, one has

$$
\int_{\Omega} \mathcal{U}_{\varepsilon}(\Phi)(x) \psi(x) d x=\frac{1}{|Y|} \int_{\Omega \times Y} \Phi(x, y) \mathcal{T}_{\varepsilon}(\psi)(x, y) d x d y .
$$

As a consequence of the duality (Hölder's inequality) and of Proposition 2.5(iv), we get the following.

Proposition 2.17. Let $p$ belong to $[1,+\infty]$. The averaging operator is linear and continuous from $L^{p}(\Omega \times Y)$ to $L^{p}(\Omega)$ and

$$
\left\|\mathcal{U}_{\varepsilon}(\Phi)\right\|_{L^{p}(\Omega)} \leq|Y|^{-\frac{1}{p}}\|\Phi\|_{L^{p}(\Omega \times Y)} .
$$

The operator $\mathcal{U}_{\varepsilon}$ maps $L^{p}(\Omega \times Y)$ into the space $L^{p}(\Omega)$. It allows one to replace the function $x \mapsto \Phi\left(x,\left\{\frac{x}{\varepsilon}\right\}_{Y}\right)$, which is meaningless, in general, by a function which always makes sense. Notice that this implies, in particular, that the largest set of test functions for two-scale convergence is actually the set $\mathcal{U}_{\varepsilon}(\Phi)$, with $\Phi$ in $L^{p^{\prime}}(\Omega \times Y)$.

It is immediate from its definition that $\mathcal{U}_{\varepsilon}$ is almost a left-inverse of $\mathcal{T}_{\varepsilon}$, since

$$
\mathcal{U}_{\varepsilon}\left(\mathcal{T}_{\varepsilon}(\phi)\right)(x)= \begin{cases}\phi(x) & \text { a.e. for } x \in \widehat{\Omega}_{\varepsilon}, \\ 0 & \text { a.e. for } x \in \Lambda_{\varepsilon},\end{cases}
$$

for every $\phi$ in $L^{p}(\Omega)$, while

$$
\mathcal{T}_{\varepsilon}\left(\mathcal{U}_{\varepsilon}(\Phi)\right)(x, y)= \begin{cases}\frac{1}{|Y|} \int_{Y} \Phi\left(\varepsilon\left[\frac{x}{\varepsilon}\right]_{Y}+\varepsilon z, y\right) d z & \text { a.e. for } \quad(x, y) \in \widehat{\Omega}_{\varepsilon} \times Y, \\ 0 & \text { a.e. for } \quad(x, y) \in \Lambda_{\varepsilon} \times Y,\end{cases}
$$

for every $\Phi$ in $L^{p}(\Omega \times Y)$. 
Proposition 2.18 (properties of $\mathcal{U}_{\varepsilon}$ ). Suppose that $p$ is in $[1,+\infty[$.

(i) Let $\left\{\Phi_{\varepsilon}\right\}$ be a bounded sequence in $L^{p}(\Omega \times Y)$ such that $\Phi_{\varepsilon} \rightarrow \Phi$ weakly in $L^{p}(\Omega \times Y)$. Then

$$
\mathcal{U}_{\varepsilon}\left(\Phi_{\varepsilon}\right) \rightarrow \mathcal{M}_{Y}(\Phi)=\frac{1}{|Y|} \int_{Y} \Phi(\cdot, y) d y \quad \text { weakly in } L^{p}(\Omega) .
$$

In particular, for every $\Phi \in L^{p}(\Omega \times Y)$,

$$
\mathcal{U}_{\varepsilon}(\Phi) \rightarrow \mathcal{M}_{Y}(\Phi) \quad \text { weakly in } L^{p}(\Omega)
$$

but not strongly, unless $\Phi$ is independent of $y$.

(ii) Let $\left\{\Phi_{\varepsilon}\right\}$ be a sequence such that $\Phi_{\varepsilon} \rightarrow \Phi$ strongly in $L^{p}(\Omega \times Y)$. Then

$$
\mathcal{T}_{\varepsilon}\left(\mathcal{U}_{\varepsilon}\left(\Phi_{\varepsilon}\right)\right) \rightarrow \Phi \quad \text { strongly in } L^{p}(\Omega \times Y) .
$$

(iii) Suppose that $\left\{w_{\varepsilon}\right\}$ is a sequence in $L^{p}(\Omega)$. Then, the following assertions are equivalent:

(a) $\mathcal{T}_{\varepsilon}\left(w_{\varepsilon}\right) \rightarrow \widehat{w}$ strongly in $L^{p}(\Omega \times Y)$,

(b) $\quad w_{\varepsilon} 1_{\widehat{\Omega}_{\varepsilon}}-\mathcal{U}_{\varepsilon}(\widehat{w}) \rightarrow 0$ strongly in $L^{p}(\Omega)$.

(iv) Suppose that $\left\{w_{\varepsilon}\right\}$ is a sequence in $L^{p}(\Omega)$. Then, the following assertions are equivalent:

(c) $\mathcal{T}_{\varepsilon}\left(w_{\varepsilon}\right) \rightarrow \widehat{w} \quad$ strongly in $L^{p}(\Omega \times Y)$ and $\int_{\Lambda_{\varepsilon}}\left|w_{\varepsilon}\right|^{p} d x \rightarrow 0$,

(d) $\quad w_{\varepsilon}-\mathcal{U}_{\varepsilon}(\widehat{w}) \rightarrow 0 \quad$ strongly in $L^{p}(\Omega)$.

Proof. (i) This follows from Proposition 2.9(ii) by duality for $p>1$. It still holds for $p=1$ in the same way as the proof of Proposition 2.9(ii). Indeed, if the De La Vallée-Poussin criterion is satisfied by the sequence $\left\{\Phi_{\varepsilon}\right\}$, it is also satisfied by the sequence $\left\{\mathcal{U}_{\varepsilon}\left(\Phi_{\varepsilon}\right)\right\}$, since for $F$ convex and continuous, Jensen's inequality implies that

$$
F\left(\mathcal{U}_{\varepsilon}\left(\Phi_{\varepsilon}\right)\right)(x) \leq \mathcal{U}_{\varepsilon}\left(F\left(\Phi_{\varepsilon}\right)\right)(x)
$$

(ii) The proof follows the same lines as that of (i)-(ii) of Proposition 2.9.

(iii) The implication $(\mathrm{a}) \Rightarrow(\mathrm{b})$ follows from (2.10) applied to $\Phi_{\varepsilon}=w_{\varepsilon} 1_{\widehat{\Omega}_{\varepsilon}}-\mathcal{U}_{\varepsilon}(\widehat{w})$ and from (2.11).

As for the converse $(\mathrm{b}) \Rightarrow(\mathrm{a})$, Proposition 2.9(ii) implies that

$$
\mathcal{T}_{\varepsilon}\left(w_{\varepsilon} 1_{\widehat{\Omega}_{\varepsilon}}-\mathcal{U}_{\varepsilon}(\widehat{w})\right) \rightarrow 0 \quad \text { strongly in } L^{p}(\Omega \times Y) .
$$

Since $\mathcal{T}_{\varepsilon}\left(w_{\varepsilon}\right)=\mathcal{T}_{\varepsilon}\left(w_{\varepsilon} 1_{\widehat{\Omega}_{\varepsilon}}\right)$, from (ii) above it converges to $\widehat{w}$ strongly in $L^{p}(\Omega \times Y)$.

(iv) The implication (c) $\Rightarrow$ (d) follows from (iii) and the second condition of (c).

Its converse $(\mathrm{d}) \Rightarrow(\mathrm{c})$ is a consequence of from (iii): since $\mathcal{U}_{\varepsilon}(\widehat{w}) 1_{\Lambda_{\varepsilon}}=0$, (d) implies (b) and $w_{\varepsilon} 1_{\Lambda_{\varepsilon}} \rightarrow 0$ in $L^{p}(\Omega)$.

Remark 2.19. The statement of Proposition 2.18(iii) does not hold with weak convergences instead of strong ones, contrary to an erroneous statement made in [24]. In view of (2.11) and Proposition 2.18(i), if $\mathcal{T}_{\varepsilon}\left(w_{\varepsilon}\right) \rightarrow \widehat{w}$ weakly in $L^{p}(\Omega \times Y)$, then $w_{\varepsilon} 1_{\widehat{\Omega}_{\varepsilon}}-\mathcal{U}_{\varepsilon}(\widehat{w})$ converges weakly to 0 in $L^{p}(\Omega)$. 
But the converse of this last implication cannot hold. Indeed, choose $\widehat{v}$ with $\mathcal{M}_{Y}(\widehat{v})=0$. By Proposition 2.18(i), $\mathcal{U}_{\varepsilon}(\widehat{v})$ converges weakly to $\mathcal{M}_{Y}(\widehat{v})=0$. Consequently, the weak limit of $w_{\varepsilon} 1_{\widehat{\Omega}_{\varepsilon}}-\mathcal{U}_{\varepsilon}(\widehat{w})$ is also the weak limit of $w_{\varepsilon} 1_{\widehat{\Omega}_{\varepsilon}}-\mathcal{U}_{\varepsilon}(\widehat{w}+\widehat{v})$. If the converse were true, it would imply that $\mathcal{T}_{\varepsilon}\left(w_{\varepsilon}\right)$ converges weakly to both $\widehat{w}$ and $\widehat{w}+\widehat{v}$. So $\widehat{v}=0$. In other words, $\mathcal{M}_{Y}(\widehat{v})=0$ would imply $\widehat{v}=0$.

Remark 2.20. Assertions (iii)(b) and (iv)(d) are corrector-type results.

Remark 2.21. The condition (iii)(a) is used by some authors to define the notion of "strong two-scale convergence." From the above considerations, condition (c) of Proposition 2.18(iv) is a better candidate for this definition.

2.3. The local average operator $\mathcal{M}_{\varepsilon}$. In this section, we consider the classical average operator associated to the partition of $\Omega$ by $\varepsilon$-cells $Y$ (setting it to be zero on the cells intersecting the boundary $\partial \Omega$ ).

Definition 2.22. The local average operator $\mathcal{M}_{\varepsilon}: L^{p}(\Omega) \mapsto L^{p}(\Omega)$, for $p \in$ $[1,+\infty]$, is defined by

$$
\mathcal{M}_{\varepsilon}(\phi)(x)=\left\{\begin{array}{l}
\frac{1}{\varepsilon^{N}|Y|} \int_{\varepsilon}\left[\frac{x}{\varepsilon}\right]_{y} \phi(\zeta) d \zeta \quad \text { if } x \in \widehat{\Omega}_{\varepsilon}, \\
0 \quad \text { if } x \in \Lambda_{\varepsilon} .
\end{array}\right.
$$

Remark 2.23. It turns out that the local average $\mathcal{M}_{\varepsilon}$ is connected to the unfolding operator $\mathcal{T}_{\varepsilon}$. Indeed, by the usual change of variable cell by cell,

$$
\mathcal{M}_{\varepsilon}(\phi)(x)=\frac{1}{|Y|} \int_{Y} \mathcal{T}_{\varepsilon}(\phi)(x, y) d y=\mathcal{M}_{Y}\left(\mathcal{I}_{\varepsilon}(\phi)\right)(x)
$$

Remark 2.24. Note that, for any $\phi$ in $L^{p}(\Omega)$, one has $\mathcal{T}_{\varepsilon}\left(\mathcal{M}_{\varepsilon}(\phi)\right)=\mathcal{M}_{\varepsilon}(\phi)$ on the set $\Omega \times Y$. Moreover, one also has $\mathcal{U}_{\varepsilon}(\phi)=\mathcal{M}_{\varepsilon}(\phi)$.

Proposition 2.25 (properties of $\mathcal{M}_{\varepsilon}$ ).

(i) Suppose that $p$ is in $[1,+\infty]$. For any any $\phi$ in $L^{p}(\Omega)$,

$$
\left\|\mathcal{M}_{\varepsilon}(\phi)\right\|_{L^{p}(\Omega)} \leq\|\phi\|_{L^{p}(\Omega)} .
$$

(ii) Suppose that $p$ is in $[1,+\infty]$. For $\phi \in L^{p}(\Omega)$ and $\psi \in L^{p^{\prime}}(\Omega)$,

$$
\int_{\Omega} \mathcal{M}_{\varepsilon}(\phi) \psi d x=\int_{\Omega} \mathcal{M}_{\varepsilon}(\phi) \mathcal{M}_{\varepsilon}(\psi) d x=\int_{\Omega} \phi \mathcal{M}_{\varepsilon}(\psi) d x
$$

(iii) Suppose that $p$ is in $\left[1,+\infty\left[\right.\right.$. Let $\left\{v_{\varepsilon}\right\}$ be a sequence such that $v_{\varepsilon} \rightarrow v$ strongly in $L^{p}(\Omega)$. Then

$$
\mathcal{M}_{\varepsilon}\left(v_{\varepsilon}\right) \rightarrow v \quad \text { strongly in } L^{p}(\Omega) .
$$

In particular, for every $\phi \in L^{p}(\Omega)$,

$$
\mathcal{M}_{\varepsilon}(\phi) \rightarrow \phi \quad \text { strongly in } L^{p}(\Omega) .
$$

(iv) Suppose that $p$ is in $\left[1,+\infty\left[\right.\right.$. Let $\left\{v_{\varepsilon}\right\}$ be a sequence such that $v_{\varepsilon} \rightarrow v$ weakly in $L^{p}(\Omega)$. Then

$$
\mathcal{M}_{\varepsilon}\left(v_{\varepsilon}\right) \rightarrow v \quad \text { weakly in } L^{p}(\Omega) .
$$

The same holds true for the weak-* topology in $L^{\infty}(\Omega)$. 
Proof. The proofs of (i) and (ii) are straightforward. The proof of (iii) is a simple consequence of (ii) of Proposition 2.9. For the proof of (iv), let $\phi$ be in $L^{p^{\prime}}(\Omega)$, with $p^{\prime} \in[1,+\infty[(p \neq 1)$, and use (2.14) and (2.15) to obtain

$$
\int_{\Omega} \phi \mathcal{M}_{\varepsilon}\left(v_{\varepsilon}\right) d x=\int_{\Omega} \mathcal{M}_{\varepsilon}(\phi) v_{\varepsilon} d x \rightarrow \int_{\Omega} \phi v d x
$$

For $p=1$, in the same way as the proof of Proposition 2.9(ii) and Proposition 2.18(i), if the De La Vallée-Poussin criterion is satisfied by the sequence $\left\{v_{\varepsilon}\right\}$, it is also satisfied by the sequence $\left\{\mathcal{M}_{\varepsilon}\left(v_{\varepsilon}\right)\right\}$, since for $F$ convex and continuous, Jensen's inequality implies that

$$
F\left(\mathcal{M}_{\varepsilon}\left(v_{\varepsilon}\right)\right)(x) \leq \mathcal{M}_{\varepsilon}\left(F\left(v_{\varepsilon}\right)\right)(x),
$$

which ends the proof.

Corollary 2.26. Suppose that $p$ is in $\left[1,+\infty\left[\right.\right.$. Let $w$ be in $L^{p}(\Omega)$ and $\left\{w_{\varepsilon}\right\}$ be a sequence in $L^{p}(\Omega)$ satisfying $\mathcal{T}_{\varepsilon}\left(w_{\varepsilon}\right) \rightarrow w$ strongly in $L^{p}(\Omega \times Y)$. Then,

$$
w_{\varepsilon} 1_{\widehat{\Omega}_{\varepsilon}} \rightarrow w \quad \text { strongly in } L^{p}(\Omega) .
$$

Furthermore, if $\int_{\Lambda_{\varepsilon}}\left|w_{\varepsilon}\right|^{p} \rightarrow 0$, then, $w_{\varepsilon} \rightarrow w$ strongly in $L^{p}(\Omega)$.

Proof. Since $w$ does not depend on $y$, one has $\mathcal{U}_{\varepsilon}(w)=\mathcal{M}_{\varepsilon}(w)$ which, by Proposition 2.25(iii), converges strongly to $w$. The conclusion follows from Proposition 2.18(iii), respectively, (iv).

3. Unfolding and gradients. This section is devoted to the properties of the restriction of the unfolding operator to the space $W^{1, p}(\Omega)$. Some results require no extra hypotheses, but many others are sensitive to the boundary conditions and the regularity of the boundary itself.

Observe that, for $w$ in $W^{1, p}(\Omega)$, one has

$$
\nabla_{y}\left(\mathcal{T}_{\varepsilon}(w)\right)=\varepsilon \mathcal{T}_{\varepsilon}(\nabla w), \quad \forall w \in W^{1, p}(\Omega) \quad \text { a.e. for } \quad(x, y) \in \Omega \times Y .
$$

Then, Proposition 2.5(iv) implies that $\mathcal{T}_{\varepsilon}$ maps $W^{1, p}(\Omega)$ into $L^{p}\left(\Omega ; W^{1, p}(Y)\right)$.

For simplicity, we assume that $Y=] 0,1\left[^{n}\right.$. Nevertheless, the results we prove here hold true in the case of a general $Y$, with minor modifications.

Proposition 3.1 (gradient in the direction of a period). Let $k$ in $[1, \ldots, n]$ and $\left\{w_{\varepsilon}\right\}$ be a bounded sequence in $L^{p}(\Omega)$, with $\left.\left.p \in\right] 1,+\infty\right]$, satisfying

$$
\varepsilon\left\|\frac{\partial w_{\varepsilon}}{\partial x_{k}}\right\|_{L^{p}(\Omega)} \leq C
$$

Then, there exist a subsequence (still denoted $\varepsilon$ ) and $\widehat{w}$ in $L^{p}(\Omega \times Y)$, with $\frac{\partial \widehat{w}}{\partial y_{k}}$ in $L^{p}(\Omega \times Y)$ such that

$$
\begin{aligned}
& \mathcal{T}_{\varepsilon}\left(w_{\varepsilon}\right) \rightarrow \widehat{w} \quad \text { weakly in } L^{p}(\Omega \times Y) \\
& \varepsilon \mathcal{T}_{\varepsilon}\left(\frac{\partial w_{\varepsilon}}{\partial x_{k}}\right)=\frac{\partial \mathcal{T}_{\varepsilon}\left(w_{\varepsilon}\right)}{\partial y_{k}} \rightarrow \frac{\partial \widehat{w}}{\partial y_{k}} \quad \text { weakly in } L^{p}(\Omega \times Y),(\text { weakly-* for } p=+\infty) .
\end{aligned}
$$

Moreover, the limit function $\widehat{w}$ is 1-periodic, with respect to the $y_{k}$ coordinate. 
Proof. Convergences (3.3) are a simple consequence of (3.1) and (3.2). It remains to prove the periodicity of $\widehat{w}$. Without loss of generality, assume $k=n$ and write $y=\left(y^{\prime}, y_{n}\right)$, with $y^{\prime}$ in $\left.Y^{\prime} \doteq\right] 0,1\left[\left[^{n-1}\right.\right.$ and $\left.y_{n} \in\right] 0,1[$.

Let $\psi \in \mathcal{D}\left(\Omega \times Y^{\prime}\right)$. Convergences (3.3) imply that the sequence $\left\{\mathcal{T}_{\varepsilon}\left(w_{\varepsilon}\right)\right\}$ is bounded in $L^{p}\left(\Omega \times Y^{\prime} ; W^{1, p}(0,1)\right)$ so that $\left\{\left.\mathcal{T}_{\varepsilon}\left(w_{\varepsilon}\right)\right|_{\left\{y_{n}=s\right\}}\right\}$ is bounded in $L^{p}\left(\Omega \times Y^{\prime}\right)$ for every $s \in[0,1]$. The periodicity with respect to $y_{n}$ results from the following computation with an obvious change of variable:

$$
\begin{aligned}
\int_{\Omega \times Y^{\prime}} & {\left[\mathcal{T}_{\varepsilon}\left(w_{\varepsilon}\right)\left(x,\left(y^{\prime}, 1\right)\right)-\mathcal{T}_{\varepsilon}\left(w_{\varepsilon}\right)\left(x,\left(y^{\prime}, 0\right)\right] \psi\left(x, y^{\prime}\right) d x d y^{\prime}\right.} \\
& =\int_{\Omega \times Y^{\prime}}\left\{w_{\varepsilon}\left(\varepsilon\left[\frac{x}{\varepsilon}\right]_{Y}+\varepsilon\left(y^{\prime}, 1\right)\right)-w_{\varepsilon}\left(\varepsilon\left[\frac{x}{\varepsilon}\right]_{Y}+\varepsilon\left(y^{\prime}, 0\right)\right)\right\} \psi\left(x, y^{\prime}\right) d x d y^{\prime} \\
& =\int_{\Omega \times Y^{\prime}} w_{\varepsilon}\left(\varepsilon\left[\frac{x}{\varepsilon}\right]_{Y}+\varepsilon\left(y^{\prime}, 0\right)\right)\left[\psi\left(x-\varepsilon e_{n}, y^{\prime}\right)-\psi\left(x, y^{\prime}\right)\right] d x d y^{\prime}, \\
& =\int_{\Omega \times Y^{\prime}} \mathcal{T}_{\varepsilon}\left(w_{\varepsilon}\right)\left(x,\left(y^{\prime}, 0\right)\right)\left[\psi\left(x-\varepsilon e_{n}, y^{\prime}\right)-\psi\left(x, y^{\prime}\right)\right] d x d y^{\prime},
\end{aligned}
$$

which goes to zero.

Corollary 3.2. Let $\left\{w_{\varepsilon}\right\}$ be in $W^{1, p}(\Omega)$, with $\left.p \in\right] 1,+\infty[$, and assume that $\left\{w_{\varepsilon}\right\}$ is a bounded sequence in $L^{p}(\Omega)$ satisfying

$$
\varepsilon\left\|\nabla w_{\varepsilon}\right\|_{L^{p}(\Omega)} \leq C .
$$

Then, there exist a subsequence (still denoted $\varepsilon$ ) and $\widehat{w} \in L^{p}\left(\Omega ; W^{1, p}(Y)\right)$ such that

$$
\begin{aligned}
& \mathcal{T}_{\varepsilon}\left(w_{\varepsilon}\right) \rightarrow \widehat{w} \quad \text { weakly in } L^{p}\left(\Omega ; W^{1, p}(Y)\right), \\
& \varepsilon \mathcal{T}_{\varepsilon}\left(\nabla w_{\varepsilon}\right) \rightarrow \nabla_{y} \widehat{w} \quad \text { weakly in } L^{p}(\Omega \times Y) .
\end{aligned}
$$

Moreover, the limit function $\widehat{w}$ is $Y$-periodic, i.e., belongs to $L^{p}\left(\Omega ; W_{\text {per }}^{1, p}(Y)\right)$, where $W_{\text {per }}^{1, p}(Y)$ denotes the Banach space of $Y$-periodic functions in $W_{l o c}^{1, p}\left(\mathbb{R}^{n}\right)$, with the $W^{1, p}(Y)$ norm.

Corollary 3.3. Let $p$ be in $] 1,+\infty\left[\right.$ and $\left\{w_{\varepsilon}\right\}$ be a sequence converging weakly in $W^{1, p}(\Omega)$ to $w$. Then,

$$
\mathcal{T}_{\varepsilon}\left(w_{\varepsilon}\right) \rightarrow w \quad \text { weakly in } L^{p}\left(\Omega ; W^{1, p}(Y)\right) .
$$

Furthermore, if $\left\{w_{\varepsilon}\right\}$ converges strongly to $w$ in $L^{p}(\Omega)$, the above convergence is strong (this is the case if, for example, $W^{1, p}(\Omega)$ is compactly embedded in $L^{p}(\Omega)$ ).

Proof. Using (3.1), since $\left\{w_{\varepsilon}\right\}$ weakly converges, one has the estimates

$$
\begin{aligned}
& \left\|\mathcal{T}_{\varepsilon}\left(w_{\varepsilon}\right)\right\|_{L^{p}(\Omega \times Y)} \leq C, \\
& \left\|\nabla_{y}\left(\mathcal{T}_{\varepsilon}\left(w_{\varepsilon}\right)\right)\right\|_{L^{p}(\Omega \times Y)} \leq \varepsilon C,
\end{aligned}
$$

so that there exist a subsequence (still denoted $\varepsilon$ ) and $\widehat{w}$ in $L^{p}\left(\Omega ; W^{1, p}(Y)\right)$ such that

$$
\begin{aligned}
& \mathcal{T}_{\varepsilon}\left(w_{\varepsilon}\right) \rightarrow \widehat{w} \quad \text { weakly in } L^{p}\left(\Omega ; W^{1, p}(Y)\right), \\
& \nabla_{y}\left(\mathcal{T}_{\varepsilon}\left(w_{\varepsilon}\right)\right) \rightarrow 0 \quad \text { strongly in } L^{p}(\Omega \times Y),
\end{aligned}
$$

and $\nabla_{y} \widehat{w}=0$. Consequently, $\widehat{w}$ does not depend on $y$, and Proposition 2.9(iii) immediately gives $w=\mathcal{M}_{Y}(\widehat{w})=\widehat{w}$. Moreover, convergence (3.4) holds for the entire 
sequence $\varepsilon$. Finally, if the sequence $\left\{w_{\varepsilon}\right\}$ converges strongly to $w$ in $L^{p}(\Omega)$, so does the sequence $\left\{\mathcal{T}_{\varepsilon}\left(w_{\varepsilon}\right)\right\}$, thanks to Proposition 2.9(ii).

Proposition 3.4. Suppose that $p$ is in $\left[1,+\infty\left[\right.\right.$. Let $\left\{w_{\varepsilon}\right\}$ be a sequence which converges strongly to some $w$ in $W^{1, p}(\Omega)$. Then,

(i) $\quad \mathcal{T}_{\varepsilon}\left(\nabla w_{\varepsilon}\right) \rightarrow \nabla w \quad$ strongly in $L^{p}(\Omega \times Y)$,

(ii) $\frac{1}{\varepsilon}\left(\mathcal{T}_{\varepsilon}\left(w_{\varepsilon}\right)-\mathcal{M}_{\varepsilon}\left(w_{\varepsilon}\right)\right) \rightarrow y^{c} \cdot \nabla w \quad$ strongly in $L^{p}\left(\Omega ; W^{1, p}(Y)\right)$,

where

$$
y^{c}=\left(y_{1}-\frac{1}{2}, \ldots, y_{n}-\frac{1}{2}\right)
$$

Proof. The first asssertion follows from Proposition 2.9(i). To prove (ii), set

$$
Z_{\varepsilon} \doteq \frac{1}{\varepsilon}\left(\mathcal{T}_{\varepsilon}\left(w_{\varepsilon}\right)-\mathcal{M}_{\varepsilon}\left(w_{\varepsilon}\right)\right)
$$

which has mean value zero in $Y$. Since

$$
\nabla_{y} Z_{\varepsilon}=\frac{1}{\varepsilon} \nabla_{y}\left(\mathcal{T}_{\varepsilon}\left(w_{\varepsilon}\right)\right)=\mathcal{T}_{\varepsilon}\left(\nabla w_{\varepsilon}\right)
$$

thanks to assertion (i),

$$
\nabla_{y} Z_{\varepsilon} \rightarrow \nabla w \quad \text { strongly in } L^{p}(\Omega \times Y) .
$$

Then recall the Poincaré-Wirtinger inequality in $Y$ :

$$
\forall \psi \in W^{1, p}(Y), \quad\left\|\psi-\mathcal{M}_{Y}(\psi)\right\|_{L^{p}(Y)} \leq C\|\nabla \psi\|_{L^{p}(Y)} .
$$

Applying it to the function $Z_{\varepsilon}-y^{c} \cdot \nabla w$ (which is of mean value zero) gives

$$
\left\|Z_{\varepsilon}-y^{c} \cdot \nabla w\right\|_{L^{p}(\Omega \times Y)} \leq C\left\|\nabla_{y} Z_{\varepsilon}-\nabla w\right\|_{L^{p}(\Omega \times Y)},
$$

and this concludes the proof.

THEOREM 3.5. Suppose that $p$ is in $] 1,+\infty\left[\right.$. Let $\left\{w_{\varepsilon}\right\}$ be a sequence converging weakly to some $w$ in $W^{1, p}(\Omega)$. Up to a subsequence, there exists some $\widehat{w}$ in $L^{p}\left(\Omega ; W_{p e r}^{1, p}(Y)\right)$ such that

(i) $\mathcal{T}_{\varepsilon}\left(\nabla w_{\varepsilon}\right) \rightarrow \nabla w+\nabla_{y} \widehat{w} \quad$ weakly in $L^{p}(\Omega \times Y)$,

(ii) $\frac{1}{\varepsilon}\left(\mathcal{T}_{\varepsilon}\left(w_{\varepsilon}\right)-\mathcal{M}_{\varepsilon}\left(w_{\varepsilon}\right)\right) \rightarrow \widehat{w}+y^{c} \cdot \nabla w$ weakly in $L^{p}\left(\Omega ; W^{1, p}(Y)\right)$.

Moreover, $\mathcal{M}_{Y}(\widehat{w})=0$.

Proof. Following the same lines as in the previous proof, introduce

$$
Z_{\varepsilon}=\frac{1}{\varepsilon}\left(\mathcal{T}_{\varepsilon}\left(w_{\varepsilon}\right)-\mathcal{M}_{\varepsilon}\left(w_{\varepsilon}\right)\right)
$$

which has mean value zero in $Y$. Since $\nabla_{y} Z_{\varepsilon}=\mathcal{T}_{\varepsilon}\left(\nabla w_{\varepsilon}\right)$, (ii) implies (i). 
To prove (ii), note that the sequence $\left\{\nabla_{y} Z_{\varepsilon}\right\}$ is bounded in $L^{p}(\Omega \times Y)$. By (3.6),

$$
\left\|Z_{\varepsilon}-y^{c} \cdot \nabla w\right\|_{L^{p}(\Omega \times Y)} \leq C
$$

so that there exists $\widehat{w}$ in $L^{p}\left(\Omega ; W^{1, p}(Y)\right)$ such that, up to a subsequence,

$$
Z_{\varepsilon}-y^{c} \cdot \nabla w \rightarrow \widehat{w} \quad \text { weakly in } L^{p}\left(\Omega ; W^{1, p}(Y)\right) .
$$

Since, by construction, $\mathcal{M}_{Y}\left(y^{c}\right)$ vanishes, so does $\mathcal{M}_{Y}(\widehat{w})$.

It remains to prove the $Y$-periodicity of $\widehat{w}$. This is obtained in the same way as in the proof of Proposition 3.1 by using a test function $\psi \in \mathcal{D}\left(\Omega \times Y^{\prime}\right)$. One has successively,

$$
\begin{aligned}
\int_{\Omega \times Y^{\prime}} & {\left[Z_{\varepsilon}\left(x,\left(y^{\prime}, 1\right)\right)-Z_{\varepsilon}\left(x,\left(y^{\prime}, 0\right)\right] \psi\left(x, y^{\prime}\right) d x d y^{\prime}\right.} \\
& =\int_{\Omega \times Y^{\prime}} \frac{1}{\varepsilon}\left\{w_{\varepsilon}\left(\varepsilon\left[\frac{x}{\varepsilon}\right]_{Y}+\varepsilon\left(y^{\prime}, 1\right)\right)-w_{\varepsilon}\left(\varepsilon\left[\frac{x}{\varepsilon}\right]_{Y}+\varepsilon\left(y^{\prime}, 0\right)\right)\right\} \psi\left(x, y^{\prime}\right) d x d y^{\prime} \\
& =\int_{\Omega \times Y^{\prime}} w_{\varepsilon}\left(\varepsilon\left[\frac{x}{\varepsilon}\right]_{Y}+\varepsilon\left(y^{\prime}, 0\right)\right) \frac{1}{\varepsilon}\left[\psi\left(x-\varepsilon e_{n}, y^{\prime}\right)-\psi\left(x, y^{\prime}\right)\right] d x d y^{\prime}, \\
& =\int_{\Omega \times Y^{\prime}} \mathcal{T}_{\varepsilon}\left(w_{\varepsilon}\right)\left(x,\left(y^{\prime}, 0\right)\right) \frac{1}{\varepsilon}\left[\psi\left(x-\varepsilon e_{n}, y^{\prime}\right)-\psi\left(x, y^{\prime}\right)\right] d x d y^{\prime} .
\end{aligned}
$$

By Proposition 2.9(ii), $\left\{\mathcal{T}_{\varepsilon}\left(w_{\varepsilon}\right)\right\}$ converges strongly to $w$ in $L^{p}(\Omega \times Y)$, and by (3.7) (i), it converges weakly to the same $w$ in $L^{p}\left(\Omega ; W^{1, p}(Y)\right)$. By the trace theorem in $W^{1, p}(Y)$, the trace of $\mathcal{T}_{\varepsilon}\left(w_{\varepsilon}\right)$ on $\Omega \times Y^{\prime}$ converges weakly to $w$ in $L^{p}\left(\Omega \times Y^{\prime}\right)$. Hence, the last integral converges to

$$
-\int_{\Omega \times Y^{\prime}} w(x) \frac{\partial \psi}{\partial x_{n}}\left(x, y^{\prime}\right) d x d y^{\prime} .
$$

Similarly, since $\left(y^{c} \cdot \nabla w\right)\left(y^{\prime}, 1\right)-\left(y^{c} \cdot \nabla w\right)\left(y^{\prime}, 0\right)=\frac{\partial w}{\partial x_{n}}$, we obtain

$$
\begin{aligned}
\int_{\Omega \times Y^{\prime}} & {\left[\left(y^{c} \cdot \nabla w\right)\left(y^{\prime}, 1\right)-\left(y^{c} \cdot \nabla w\right)\left(y^{\prime}, 0\right)\right] \psi\left(x, y^{\prime}\right) d x d y^{\prime} } \\
= & \int_{\Omega \times Y^{\prime}} \frac{\partial w}{\partial x_{n}} \psi\left(x, y^{\prime}\right) d x d y^{\prime}=-\int_{\Omega \times Y^{\prime}} w(x) \frac{\partial \psi}{\partial x_{n}}\left(x, y^{\prime}\right) d x d y^{\prime} .
\end{aligned}
$$

This, together with (3.8) and convergence (3.7)(ii), shows that

$$
\int_{\Omega \times Y^{\prime}}\left[\widehat{w}\left(x,\left(y^{\prime}, 1\right)\right)-\widehat{w}\left(x,\left(y^{\prime}, 0\right)\right] \psi\left(x, y^{\prime}\right) d x d y^{\prime}=0,\right.
$$

so that $\widehat{w}$ is $y_{n}$-periodic. The same holds in the directions of all of the other periods.

Theorem 3.5 can be generalized to the case of $W^{k, p}(\Omega)$-spaces, with $k \geq 1$ and $p \in] 1,+\infty\left[\right.$. In order to do so, for $r=\left(r_{1}, \ldots, r_{n}\right) \in \mathbb{N}^{n}$ with $|r|=r_{1}+\cdots+r_{n} \leq k$, introduce the notation $D^{r}$ and $D_{y}^{r}$ :

$$
D^{r}=\frac{\partial^{|r|}}{\partial x_{1}^{r_{1}} \ldots \partial x_{n}^{r_{n}}}, \quad D_{y}^{r}=\frac{\partial^{|r|}}{\partial y_{1}^{r_{1}} \ldots \partial y_{n}^{r_{n}}} .
$$

Then the following result holds. 
THEOREM 3.6. Let $\left\{w_{\varepsilon}\right\}$ be a sequence converging weakly in $W^{k, p}(\Omega)$ to $w$, $k \geq 1$, and $p \in] 1,+\infty[$. There exist a subsequence (still denoted $\varepsilon$ ) and $\widehat{w}$ in the space $L^{p}\left(\Omega ; W_{\text {per }}^{k, p}(Y)\right)$ such that

$$
\begin{cases}\mathcal{T}_{\varepsilon}\left(D^{l} w_{\varepsilon}\right) \rightarrow D^{l} w \quad \text { weakly in } L^{p}\left(\Omega ; W^{k-l, p}(Y)\right), & |l| \leq k-1, \\ \mathcal{T}_{\varepsilon}\left(D^{l} w_{\varepsilon}\right) \rightarrow D^{l} w+D_{y}^{l} \widehat{w} \quad \text { weakly in } L^{p}(\Omega \times Y), & |l|=k .\end{cases}
$$

Furthermore, if $\left\{w_{\varepsilon}\right\}$ converges strongly to $w$ in $W^{k-1, p}(\Omega)$, the above convergences are strong in $L^{p}\left(\Omega ; W^{k-l, p}(Y)\right)$ for $|l| \leq k-1$.

Proof. We give a brief proof for $k=2$. The same argument generalizes for $k>2$. If $|l|=1$, the first convergence in (3.9) follows directly from Corollary 3.3. Set

$$
W_{\varepsilon}=\frac{1}{\varepsilon^{2}}\left[\mathcal{T}_{\varepsilon}\left(w_{\varepsilon}\right)-\mathcal{M}_{\varepsilon}\left(w_{\varepsilon}\right)-y^{c} \cdot \mathcal{M}_{\varepsilon}\left(\nabla w_{\varepsilon}\right)\right] .
$$

The sequence $\left\{w_{\varepsilon}\right\}$ is bounded in $W^{2, p}(\Omega)$, hence proceeding as in the proof of Proposition 2.25(iii), one obtains

$$
\left\|W_{\varepsilon}\right\|_{L^{p}(\Omega \times Y)} \leq C
$$

Moreover,

$$
\nabla_{y}\left(W_{\varepsilon}\right)=\frac{1}{\varepsilon^{2}}\left(\mathcal{T}_{\varepsilon}\left(\nabla w_{\varepsilon}\right)-\mathcal{M}_{\varepsilon}\left(\nabla w_{\varepsilon}\right)\right)
$$

and

$$
D_{y}^{l}\left(W_{\varepsilon}\right)=\mathcal{T}_{\varepsilon}\left(D^{l} w_{\varepsilon}\right), \quad \text { with } \quad|l|=2 .
$$

This implies that the sequence $\left\{W_{\varepsilon}\right\}$ is bounded in $L^{p}\left(\Omega ; W^{2, p}(Y)\right)$. Therefore, there exist a subsequence (still denoted $\varepsilon$ ) and $\widetilde{w} \in L^{p}\left(\Omega ; W^{2, p}(Y)\right)$ such that

$$
\begin{aligned}
& W_{\varepsilon} \rightarrow \widetilde{w} \quad \text { weakly in } L^{p}\left(\Omega ; W^{2, p}(Y)\right), \\
& \frac{\partial W_{\varepsilon}}{\partial y_{i}}=\frac{1}{\varepsilon^{2}}\left(\mathcal{T}_{\varepsilon}\left(\frac{\partial w_{\varepsilon}}{\partial x_{i}}\right)-\mathcal{M}_{\varepsilon}\left(\frac{\partial w_{\varepsilon}}{\partial x_{i}}\right)\right) \rightarrow \frac{\partial \widetilde{w}}{\partial y_{i}} \quad \text { weakly in } L^{p}\left(\Omega ; W^{1, p}(Y)\right) .
\end{aligned}
$$

Consequently,

$$
D_{y}^{l}\left(W_{\varepsilon}\right)=\mathcal{T}_{\varepsilon}\left(D^{l} w_{\varepsilon}\right) \rightarrow D_{y}^{l} \widetilde{w} \quad \text { weakly in } L^{p}(\Omega \times Y), \quad|l|=2 .
$$

Now, apply Theorem 3.5 to each of the derivatives $\frac{\partial w_{\varepsilon}}{\partial x_{i}}, i \in\{1, \ldots, n\}$. There exist a subsequence (still denoted $\varepsilon$ ) and $\widehat{w}_{i} \in L^{p}\left(\Omega ; W_{p e r}^{1, p}(Y)\right)$ such that $\mathcal{M}_{Y}\left(\widehat{w}_{i}\right) \equiv 0$ and

$$
\frac{1}{\varepsilon}\left(\mathcal{T}_{\varepsilon}\left(\frac{\partial w_{\varepsilon}}{\partial x_{i}}\right)-\mathcal{M}_{\varepsilon}\left(\frac{\partial w_{\varepsilon}}{\partial x_{i}}\right)\right) \rightarrow y^{c} \cdot \nabla \frac{\partial w}{\partial x_{i}}+\widehat{w}_{i} \quad \text { weakly in } L^{p}(\Omega \times Y) .
$$

Then (3.10) gives

$$
\forall i \in\{1, \ldots, n\}, \quad \frac{\partial \widetilde{w}}{\partial y_{i}}=y^{c} \cdot \nabla \frac{\partial w}{\partial x_{i}}+\widehat{w}_{i} .
$$

Set

$$
\widehat{w}=\widetilde{w}-\frac{1}{2} \sum_{i, j=1}^{n}\left(y_{i}^{c} y_{j}^{c}-\mathcal{M}_{Y}\left(y_{i}^{c} y_{j}^{c}\right)\right) \frac{\partial^{2} w}{\partial x_{i} \partial x_{j}} .
$$


By construction, the function $\widehat{w}$ belongs to $L^{p}\left(\Omega ; W^{2, p}(Y)\right)$. Furthermore,

$$
\mathcal{M}_{Y}(\widehat{w})=0, \quad \frac{\partial \widehat{w}}{\partial y_{i}}=\frac{\partial \widetilde{w}}{\partial y_{i}}-y^{c} \cdot \nabla\left(\frac{\partial w}{\partial x_{i}}\right)=\widehat{w}_{i}, \quad \text { and } \quad \mathcal{M}_{Y}\left(\nabla_{y} \widehat{w}\right)=0 .
$$

The last equality implies that $\widehat{w}$ belongs to $L^{p}\left(\Omega ; W_{\text {per }}^{2, p}(Y)\right)$. Finally from (3.12) one gets

$$
D_{y}^{l} \widetilde{w}=D^{l} w+D_{y}^{l} \widehat{w}, \quad \text { with } \quad|l|=2,
$$

which together with (3.11), proves the last convergence of (3.9).

COROLlarY 3.7. Let $\left\{w_{\varepsilon}\right\}$ be a sequence converging weakly in $W^{2, p}(\Omega)$ to $w$, and $p \in] 1,+\infty[$. Then, there exist a subsequence (still denoted $\varepsilon$ ) and $\widehat{w}$ in the space $L^{p}\left(\Omega ; W_{\text {per }}^{2, p}(Y)\right)$ such that

$$
\frac{1}{\varepsilon^{2}}\left[\mathcal{T}_{\varepsilon}\left(w_{\varepsilon}\right)-\mathcal{M}_{\varepsilon}\left(w_{\varepsilon}\right)-y^{c} \cdot \mathcal{M}_{\varepsilon}\left(\nabla w_{\varepsilon}\right)\right] \rightarrow \frac{1}{2} \sum_{i, j=1}^{n}\left(y_{i}^{c} y_{j}^{c}-\mathcal{M}_{Y}\left(y_{i}^{c} y_{j}^{c}\right)\right) \frac{\partial^{2} w}{\partial x_{i} \partial x_{j}}+\widehat{w}
$$

weakly in $L^{p}\left(\Omega ; W^{2, p}(Y)\right)$, where $\widehat{w}$ is such that $\mathcal{M}_{Y}(\widehat{w})=0$.

Remark 3.8. For the case $Y=] 0,1\left[{ }^{n}, y^{c}\right.$ was defined in Proposition 3.4. For a general $Y$, all of the statements of this section hold true, with $y^{c}=y-\mathcal{M}_{Y}(y)$.

4. Macro-micro decomposition: The scale-splitting operators $\mathcal{Q}_{\varepsilon}$ and $\boldsymbol{\mathcal { R }}_{\boldsymbol{\varepsilon}}$. In this section, we give a different proof of Theorem 3.5, which was the one given originally in [24]. It is based on a scale-separation decomposition which is useful in some specific situations, for example, in the statement of general corrector results (see section 6).

The procedure is based on a splitting of functions $\phi$ in $W^{1, p}(\Omega)$ (or in $W_{0}^{1, p}(\Omega)$ ) for $p \in[1,+\infty]$, in the form

$$
\phi=\mathcal{Q}_{\varepsilon}(\phi)+\mathcal{R}_{\varepsilon}(\phi)
$$

where $\mathcal{Q}_{\varepsilon}(\phi)$ is an approximation of $\phi$ having the same behavior as $\phi$, while $\mathcal{R}_{\varepsilon}(\phi)$ is a remainder of order $\varepsilon$. Applied to the sequence $\left\{w_{\varepsilon}\right\}$ converging weakly to $w$ in $W^{1, p}(\Omega)$, it shows that, while $\left\{\nabla w_{\varepsilon}\right\},\left\{\nabla\left(\mathcal{Q}_{\varepsilon}\left(w_{\varepsilon}\right)\right)\right\}$ and $\left\{\mathcal{T}_{\varepsilon}\left(\nabla \mathcal{Q}_{\varepsilon}\left(w_{\varepsilon}\right)\right)\right\}$ have the same weak limit $\nabla w$ in $L^{p}(\Omega)$, respectively, in $L^{p}(\Omega \times Y)$, the sequence $\mathcal{T}_{\varepsilon}\left(\nabla\left(\mathcal{R}_{\varepsilon}\left(w_{\varepsilon}\right)\right)\right)$ converges weakly in $L^{p}(\Omega \times Y)$ to $\nabla_{y} \widehat{w}^{\prime}$ for some $\widehat{w}^{\prime}$ in $L^{p}\left(\Omega ; W_{p e r}^{1, p}(Y)\right)$.

We will distinguish between the case $W_{0}^{1, p}(\Omega)$ and the case $W^{1, p}(\Omega)$. For the former, any function $\phi$ in $W_{0}^{1, p}(\Omega)$ is extended by zero to the whole of $\mathbb{R}^{n}$, and this extension is denoted by $\widetilde{\phi}$. In the latter case, we suppose that $\partial \Omega$ is smooth enough so that there exists a continuous extension operator $\mathcal{P}: W^{1, p}(\Omega) \mapsto W^{1, p}\left(\mathbb{R}^{n}\right)$ satisfying

$$
\|\mathcal{P}(\phi)\|_{W^{1, p}\left(\mathbb{R}^{n}\right)} \leq C\|\phi\|_{W^{1, p}(\Omega)}, \quad \forall \phi \in W^{1, p}(\Omega),
$$

where $C$ is a constant depending on $p$ and $\partial \Omega$ only.

The construction of $\mathcal{Q}_{\varepsilon}$ is based on the $Q_{1}$ interpolate of some discrete approximation, as is customary in FEM. The idea of using these types of interpolate was already present in Griso [40], [41] for the study of truss-like structures. For the purpose of this paper, it is enough to take the average on $\varepsilon \xi+\varepsilon Y$ to construct the discrete approximations, but the average on $\varepsilon \xi+\varepsilon Y^{\prime}$, where $Y^{\prime}$ is any fixed open subset of $Y$, 
or any open subset of a manifold of codimension 1 in $Y$. The only property which is needed is the Poincaré-Wirtinger inequality, which holds in both of these cases.

Definition 4.1. The operator $\mathcal{Q}_{\varepsilon}: L^{p}\left(\mathbb{R}^{n}\right) \mapsto W^{1, \infty}\left(\mathbb{R}^{n}\right)$, for $p \in[1,+\infty]$, is defined as follows:

$$
\mathcal{Q}_{\varepsilon}(\phi)(\varepsilon \xi)=\mathcal{M}_{\varepsilon}(\phi)(\varepsilon \xi) \quad \text { for } \xi \in \varepsilon \mathbb{Z}^{n}
$$

and for any $x \in \mathbb{R}^{n}$, we set

$$
\begin{aligned}
& \mathcal{Q}_{\varepsilon}(\phi)(x) \text { is the } Q_{1} \text { interpolate of the values of } \mathcal{Q}_{\varepsilon}(\phi) \text { at the vertices } \\
& \text { of the cell } \varepsilon\left[\frac{x}{\varepsilon}\right]_{Y}+\varepsilon Y \text {. }
\end{aligned}
$$

In the case of the space $W_{0}^{1, p}(\Omega)$, the operator $\mathcal{Q}_{\varepsilon}: W_{0}^{1, p}(\Omega) \mapsto W^{1, \infty}(\Omega)$ is defined by

$$
\mathcal{Q}_{\varepsilon}(\phi)=\left.\mathcal{Q}_{\varepsilon}(\widetilde{\phi})\right|_{\Omega}
$$

where $\mathcal{Q}_{\varepsilon}(\widetilde{\phi})$ is given by (4.1).

In the case of the space $W^{1, p}(\Omega)$, the operator $\mathcal{Q}_{\varepsilon}: W^{1, p}(\Omega) \mapsto W^{1, \infty}(\Omega)$ is defined by

$$
\mathcal{Q}_{\varepsilon}(\phi)=\left.\mathcal{Q}_{\varepsilon}(\mathcal{P}(\phi))\right|_{\Omega},
$$

where $\mathcal{Q}_{\varepsilon}(\mathcal{P}(\phi))$ is given by (4.1).

We start with the following estimates.

Proposition 4.2 (properties of $\mathcal{Q}_{\varepsilon}$ on $\left.\mathbb{R}^{n}\right)$. For $\phi$ in $L^{p}\left(\mathbb{R}^{n}\right), p \in[1,+\infty]$, there exists a constant $C$ depending on $n$ and $Y$ only, such that
(i) $\left\|\mathcal{Q}_{\varepsilon}(\phi)\right\|_{L^{p}\left(\mathbb{R}^{n}\right)} \leq C\|\phi\|_{L^{p}\left(\mathbb{R}^{n}\right)}$,
(ii) $\left\|\nabla \mathcal{Q}_{\varepsilon}(\phi)\right\|_{L^{p}\left(\mathbb{R}^{n}\right)} \leq \frac{C}{\varepsilon}\|\phi\|_{L^{p}\left(\mathbb{R}^{n}\right)}$,
(iii) $\left\|\mathcal{Q}_{\varepsilon}(\phi)\right\|_{L^{\infty}\left(\mathbb{R}^{n}\right)} \leq \frac{C}{\varepsilon^{n / p}}\|\phi\|_{L^{p}\left(\mathbb{R}^{n}\right)}$,
(iv) $\left\|\nabla \mathcal{Q}_{\varepsilon}(\phi)\right\|_{L^{\infty}\left(\mathbb{R}^{n}\right)} \leq \frac{C}{\varepsilon^{1+n / p}}\|\phi\|_{L^{p}\left(\mathbb{R}^{n}\right)}$.

Furthermore, for any $\psi$ in $L^{p}(Y)$,

$$
\left\|\mathcal{Q}_{\varepsilon}(\phi) \psi\left(\left\{\frac{\cdot}{\varepsilon}\right\}_{Y}\right)\right\|_{L^{p}\left(\mathbb{R}^{n}\right)} \leq C\|\phi\|_{L^{p}\left(\mathbb{R}^{n}\right)}\|\psi\|_{L^{p}(Y)} ;
$$

if $\psi$ is in $W_{p e r}^{1, p}(Y)$, then

$$
\left\|\mathcal{Q}_{\varepsilon}(\phi) \psi\left(\left\{\frac{\dot{c}}{\varepsilon}\right\}_{Y}\right)\right\|_{W^{1, p}\left(\mathbb{R}^{n}\right)} \leq \frac{C}{\varepsilon}\|\phi\|_{L^{p}\left(\mathbb{R}^{n}\right)}\|\psi\|_{W^{1, p}(Y)} .
$$

Proof. By definition, the $Q_{1}$ interpolate is Lipschitz-continuous and reaches its maximum at some $\varepsilon \xi$. So, to estimate the $L^{\infty}$ norm of $\mathcal{Q}_{\varepsilon}(\phi)$, it suffices to estimate the $\mathcal{Q}_{\varepsilon}(\phi)(\varepsilon \xi)^{\prime} s$. By $(4.1)$,

$$
\left|\mathcal{Q}_{\varepsilon}(\phi)(\varepsilon \xi)\right|^{p} \leq \frac{1}{|Y|} \int_{Y}|\phi(\varepsilon \xi+\varepsilon z)|^{p} d z=\frac{1}{\varepsilon^{n}|Y|} \int_{\varepsilon \xi+\varepsilon Y}|\phi(x)|^{p} d x .
$$

Since

$$
\frac{1}{\varepsilon^{n}|Y|} \int_{\varepsilon \xi+\varepsilon Y}|\phi(x)|^{p} d x \leq \frac{1}{\varepsilon^{n}|Y|}\|\phi\|_{L^{p}\left(\mathbb{R}^{n}\right)}^{p},
$$

estimate (iii) follows, with $C=\frac{1}{|Y|^{1 / p}}$. 
The space $Q_{1}(Y)$ is of dimension $2^{n}$, hence all of the norms are equivalent. So, there are constants $c_{1}, c_{2}$, and $c_{3}$ (depending only upon $p$ and $Y$ ) such that, for every $\Phi \in Q_{1}(Y)$

$$
\begin{aligned}
\|\nabla \Phi\|_{L^{\infty}(Y)} \leq c_{1} \sum_{\kappa \in\{0,1\}^{n}}\left|\Phi\left(\sum_{j=1}^{n} \kappa_{j} b_{j}\right)\right|, \\
\|\Phi\|_{L^{p}(Y)} \leq c_{2}\left(\sum_{\kappa \in\{0,1\}^{n}}\left|\Phi\left(\sum_{j=1}^{n} \kappa_{j} b_{j}\right)\right|^{p}\right)^{1 / p}, \\
\|\nabla \Phi\|_{L^{p}(Y)} \leq c_{3}\left(\sum_{\kappa \in\{0,1\}^{n}}\left|\Phi\left(\sum_{j=1}^{n} \kappa_{j} b_{j}\right)\right|^{p}\right)^{1 / p} .
\end{aligned}
$$

Rescaling these inequalities for $\Phi(y) \doteq \mathcal{Q}_{\varepsilon}(\phi)(\varepsilon \xi+\varepsilon y)$, gives

$$
\begin{aligned}
\left\|\nabla \mathcal{Q}_{\varepsilon}(\phi)\right\|_{L^{\infty}(\varepsilon \xi+\varepsilon Y)} & \leq \frac{c_{1}}{\varepsilon} \sum_{\kappa \in\{0,1\}^{n}}\left|\mathcal{Q}_{\varepsilon}(\phi)\left(\varepsilon \xi+\varepsilon \sum_{j=1}^{n} \kappa_{j} b_{j}\right)\right|, \\
\left\|\mathcal{Q}_{\varepsilon}(\phi)\right\|_{L^{p}(\varepsilon \xi+\varepsilon Y)} & \leq c_{2} \varepsilon^{n / p}\left(\sum_{\kappa \in\{0,1\}^{n}}\left|\mathcal{Q}_{\varepsilon}(\phi)\left(\varepsilon \xi+\varepsilon \sum_{j=1}^{n} \kappa_{j} b_{j}\right)\right|^{p}\right)^{1 / p}, \\
\left\|\nabla \mathcal{Q}_{\varepsilon}(\phi)\right\|_{L^{p}(\varepsilon \xi+\varepsilon Y)} & \leq c_{3} \varepsilon^{n / p-1}\left(\sum_{\kappa \in\{0,1\}^{n}}\left|\mathcal{Q}_{\varepsilon}(\phi)\left(\varepsilon \xi+\varepsilon \sum_{j=1}^{n} \kappa_{j} b_{j}\right)\right|^{p}\right)^{1 / p} .
\end{aligned}
$$

Using (4.5), we have

$$
\left\|\nabla \mathcal{Q}_{\varepsilon}(\phi)\right\|_{L^{\infty}\left(\mathbb{R}^{n}\right)} \leq \frac{2^{n} c_{1}}{\varepsilon^{1+n / p}|Y|^{1 / p}}\|\phi\|_{L^{p}\left(\mathbb{R}^{n}\right)},
$$

which gives (iv). Similarly,

$$
\left\|\mathcal{Q}_{\varepsilon}(\phi)\right\|_{L^{p}(\varepsilon \xi+\varepsilon Y)}^{p} \leq \frac{c_{2}^{p}}{|Y|} \sum_{\kappa \in\{0,1\}^{n}} \int_{\varepsilon \xi+\varepsilon \sum_{j=1}^{n} \kappa_{j} b_{j}+\varepsilon Y}|\phi(x)|^{p} d x
$$

which, by summation on $\xi \in \Xi_{\varepsilon}$, gives (i), with $C=\frac{\left(2 c_{2}\right)^{n / p}}{|Y|^{1 / p}}$.

Estimate (ii), with $C=\frac{\left(2 c_{3}\right)^{n / p}}{|Y|^{1 / p}}$, follows by a similar computation.

To prove (4.3), observe first that the function $\mathcal{Q}_{\varepsilon}(\phi) \psi\left(\{\dot{\bar{\varepsilon}}\}_{Y}\right)$ belongs to $L^{p}\left(\mathbb{R}^{n}\right)$, since $\mathcal{Q}_{\varepsilon}(\phi)$ is in $L^{\infty}\left(\mathbb{R}^{n}\right)$ and $\psi\left(\{\dot{\bar{\varepsilon}}\}_{Y}\right)$ is in $L^{p}\left(\mathbb{R}^{n}\right)$. Moreover,

$$
\left\|\psi\left(\left\{\frac{\dot{\varepsilon}}{\varepsilon}\right\}_{Y}\right)\right\|_{L^{p}(\varepsilon \xi+\varepsilon Y)}^{p}=\varepsilon^{n}\|\psi\|_{L^{p}(Y)}^{p},
$$

while, by (4.5),

$$
\left\|\mathcal{Q}_{\varepsilon}(\phi)\right\|_{L^{\infty}(\varepsilon \xi+\varepsilon Y)}^{p} \leq \sum_{\kappa \in\{0,1\}^{n}} \frac{1}{\varepsilon^{n}|Y|} \int_{\varepsilon \xi+\varepsilon Y+\varepsilon \sum_{j=1}^{n} \kappa_{j} b_{j}}|\phi(x)|^{p} .
$$

Using these two estimates and summing on $\Xi_{\varepsilon}$ gives (4.3), with $C=\frac{2^{n / p}}{|Y|^{1 / p}}$. 
Estimate (4.4) is obtained in a similar fashion, with $C=\frac{(2)^{n / p}+\left(2 c_{3}\right)^{n / p}}{|Y|^{1 / p}}$.

Corollary 4.3. For $\phi$ in $L^{p}\left(\mathbb{R}^{n}\right), p \in[1,+\infty[$, the following convergences hold: $\mathcal{Q}_{\varepsilon}(\phi) \rightarrow \phi \quad$ strongly in $L^{p}\left(\mathbb{R}^{n}\right)$, $\varepsilon \nabla \mathcal{Q}_{\varepsilon}(\phi) \rightarrow 0 \quad$ strongly in $\left(L^{p}\left(\mathbb{R}^{n}\right)\right)^{n}$.

DeFinition 4.4. The remainder $\mathcal{R}_{\varepsilon}(\phi)$ is given by

$$
\mathcal{R}_{\varepsilon}(\phi)=\phi-\mathcal{Q}_{\varepsilon}(\phi) \quad \text { for any } \phi \in W^{1, p}(\Omega) .
$$

The following proposition is well-known from the FEM.

Proposition 4.5 (properties of $\mathcal{Q}_{\varepsilon}$ and $\mathcal{R}_{\varepsilon}$ ). For the case $W_{0}^{1, p}(\Omega)$, one has

$$
\begin{aligned}
\text { (i) } & \left\|\mathcal{Q}_{\varepsilon}(\phi)\right\|_{W^{1, p}(\Omega)} \leq C\|\phi\|_{W_{0}^{1, p}(\Omega)}, \\
\text { (ii) } & \left\|\mathcal{R}_{\varepsilon}(\phi)\right\|_{L^{p}(\Omega)} \leq \varepsilon C\|\phi\|_{W_{0}^{1, p}(\Omega)}, \\
\text { (iii) } & \left\|\nabla \mathcal{R}_{\varepsilon}(\phi)\right\|_{L^{p}(\Omega)} \leq C\|\nabla \phi\|_{L^{p}(\Omega)} .
\end{aligned}
$$

The constant $C$ depends on $Y$ (via its diameter and its Poincaré-Wirtinger constant) only, and depends neither on $\Omega$ nor on $\varepsilon$.

Similarly, for the case $W^{1, p}(\Omega)$, one has

$$
\begin{aligned}
& \text { (iv) }\left\|\mathcal{Q}_{\varepsilon}(\phi)\right\|_{W^{1, p}(\Omega)} \leq C\|\mathcal{P}\|\|\phi\|_{W^{1, p}(\Omega)}, \\
& \text { (v) }\left\|\mathcal{R}_{\varepsilon}(\phi)\right\|_{L^{p}(\Omega)} \leq \varepsilon C\|\mathcal{P}\|\|\phi\|_{W^{1, p}(\Omega)}, \\
& \text { (vi) }\left\|\nabla \mathcal{R}_{\varepsilon}(\phi)\right\|_{L^{p}(\Omega)} \leq C\|\mathcal{P}\|\|\nabla \phi\|_{L^{p}(\Omega)} .
\end{aligned}
$$

Moreover, in both cases,

$$
\left\|\frac{\partial^{2} \mathcal{Q}_{\varepsilon}(\phi)}{\partial x_{i} \partial x_{j}}\right\|_{L^{p}(\Omega)} \leq \frac{C^{\prime}}{\varepsilon}\|\nabla \phi\|_{L^{p}(\Omega)} \quad \text { for } i, j \in[1, \ldots, n], \quad i \neq j,
$$

where $C^{\prime}$ does not depend on $\varepsilon$.

Proof. We start with $\phi$ in $W^{1, p}\left(\mathbb{R}^{n}\right)$. From Proposition 2.5(i) and inequality (3.5), we get

$$
\left\|\phi-\mathcal{M}_{\varepsilon}(\phi)\right\|_{L^{p}\left(\mathbb{R}^{n}\right)}=|Y|^{-\frac{1}{p}}\left\|\mathcal{T}_{\varepsilon}(\phi)-\mathcal{M}_{\varepsilon}(\phi)\right\|_{L^{p}\left(\mathbb{R}^{n} \times Y\right)} \leq \varepsilon C\|\nabla \phi\|_{L^{p}\left(\mathbb{R}^{n}\right)} .
$$
have

On the other hand, for any $\psi \in W^{1, p}\left(\operatorname{interior}\left(\overline{Y \cup\left(Y+e_{i}\right)}\right)\right), \quad i \in\{1, \ldots, n\}$, we

$$
\begin{aligned}
& \left|\mathcal{M}_{Y+e_{i}}(\psi)-\mathcal{M}_{Y}(\psi)\right|=\left|\mathcal{M}_{Y}\left(\psi\left(\cdot+e_{i}\right)-\psi(\cdot)\right)\right| \\
& \leq\left\|\psi\left(\cdot+e_{i}\right)-\psi(\cdot)\right\|_{L^{p}(Y)} \leq C\|\nabla \psi\|_{L^{p}\left(Y \cup\left(Y+e_{i}\right)\right)}
\end{aligned}
$$

By a scaling argument and using Definition 4.1, this gives

$$
\left|\mathcal{Q}_{\varepsilon}(\phi)(\varepsilon \xi)-\mathcal{Q}_{\varepsilon}(\phi)\left(\varepsilon \xi+\varepsilon e_{i}\right)\right| \leq \varepsilon C\|\nabla \phi\|_{L^{p}}\left(\varepsilon(\xi+Y) \cup \varepsilon\left(\xi+e_{i}+Y\right)\right)
$$

for all $\xi \in \varepsilon \mathbb{Z}^{n}$.

Let $x \in \varepsilon(\xi+Y)$, and set for every $\kappa=\left(\kappa_{1}, \ldots, \kappa_{n}\right) \in\{0,1\}^{n}$,

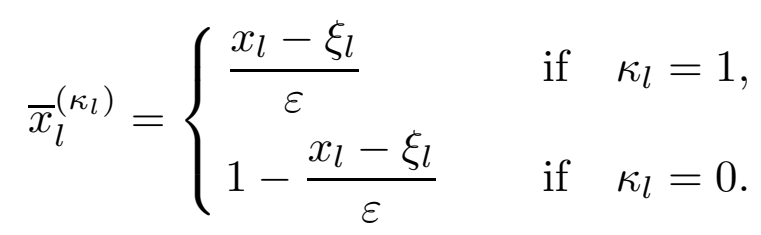


If $\xi \in \varepsilon \mathbb{Z}^{n}$, for every $\kappa \in\{0,1\}^{n}$, by definition we have

$$
\mathcal{Q}_{\varepsilon}(\phi)(x)=\sum_{\kappa \in\{0,1\}^{n}} \mathcal{Q}_{\varepsilon}(\phi)(\varepsilon \xi+\varepsilon \kappa) \bar{x}_{1}^{\left(\kappa_{1}\right)} \ldots \bar{x}_{n}^{\left(\kappa_{n}\right)}
$$

and so, for example,

$$
\begin{aligned}
& \frac{\partial \mathcal{Q}_{\varepsilon}(\phi)}{\partial x_{1}}(x) \\
& =\sum_{\kappa_{2}, \ldots, \kappa_{n}} \frac{\mathcal{Q}_{\varepsilon}(\phi)\left(\varepsilon \xi+\varepsilon\left(1, \kappa_{2}, \ldots, \kappa_{n}\right)\right)-\mathcal{Q}_{\varepsilon}(\phi)\left(\varepsilon \xi+\varepsilon\left(0, \kappa_{2}, \ldots, \kappa_{n}\right)\right)}{\varepsilon} \bar{x}_{2}^{\left(\kappa_{2}\right)} \ldots \bar{x}_{n}^{\left(\kappa_{n}\right)},
\end{aligned}
$$

and a same expression for the other derivatives. This last formula and (4.7)-(4.9) imply estimate (i) written in $\mathbb{R}^{n}$.

Now, from (4.9), we get

$$
\phi(x)-\mathcal{Q}_{\varepsilon}(\phi)(x)=\sum_{\kappa \in\{0,1\}^{n}}\left(\phi(x)-\mathcal{Q}_{\varepsilon}(\phi)(\varepsilon \xi+\varepsilon \kappa)\right) \bar{x}_{1}^{\left(\kappa_{1}\right)} \ldots \bar{x}_{n}^{\left(\kappa_{n}\right)},
$$

and (ii) (in $\mathbb{R}^{n}$ ) follows by using estimate (4.7). Estimate (iii) (again in $\mathbb{R}^{n}$ ) is straightforward from the previous ones.

If $\phi$ is in $W_{0}^{1, p}(\Omega)$, let $\widetilde{\phi}$ be its extension to the whole of $\mathbb{R}^{n}$. To derive (i)-(iii), it suffices to write down the estimates in $\mathbb{R}^{n}$ obtained above. Similarly, applying them to $\mathcal{P}(\phi)$ for $\phi$ in $W^{1, p}(\Omega)$ gives (iv)-(vi).

To finish the proof, it remains to show estimate (4.6). To do so, it is enough to take the derivative with respect to any $x_{k}$, with $k \neq 1$ in the formula of $\frac{\partial \mathcal{Q}_{\varepsilon}(\phi)}{\partial x_{1}}$ above, and use estimate (4.8).

Remark 4.6. By construction (see explicit formula (4.9)), the function $\mathcal{Q}_{\varepsilon}(\phi)$ is separately piecewise linear on each cell. Observe also that, for any $k \in\{1, \ldots, n\}$, $\frac{\partial \mathcal{Q}_{\varepsilon}(\phi)}{\partial x_{k}}$ is independent of $x_{k}$ in each cell $\varepsilon(\xi+Y)$.

Proposition 4.7. Let $\left\{w_{\varepsilon}\right\}$ be a sequence converging weakly in $W_{0}^{1, p}(\Omega)$ (resp. $\left.W^{1, p}(\Omega)\right)$ to $w$. Then, the following convergences hold:

$$
\begin{aligned}
\text { (i) } & \mathcal{R}_{\varepsilon}\left(w_{\varepsilon}\right) \rightarrow 0 \quad \text { strongly in } L^{p}(\Omega), \\
\text { (ii) } & \mathcal{Q}_{\varepsilon}\left(w_{\varepsilon}\right) \rightarrow w \quad \text { weakly in } W^{1, p}(\Omega), \\
\text { (iii) } & \mathcal{T}_{\varepsilon}\left(\nabla \mathcal{Q}_{\varepsilon}\left(w_{\varepsilon}\right)\right) \rightarrow \nabla w \quad \text { weakly in } L^{p}(\Omega \times Y) .
\end{aligned}
$$

Proof. Convergence (i) is a direct consequence of estimate (ii) (resp. (v)) of Proposition 4.5, and it implies convergence (ii). Together with (i), Proposition 2.9(ii) implies $\mathcal{T}_{\varepsilon}\left(\mathcal{Q}_{\varepsilon}\left(w_{\varepsilon}\right)\right) \rightarrow w \quad$ weakly in $L^{p}(\Omega \times Y)$. From (4.5),

$$
\left\|\frac{\partial}{\partial x_{i}}\left(\frac{\partial \mathcal{Q}_{\varepsilon}\left(w_{\varepsilon}\right)}{\partial x_{j}}\right)\right\|_{L^{p}(\Omega)} \leq \frac{C}{\varepsilon} \quad \text { for } i, j \in[1, \ldots, n], \quad i \neq j .
$$

Now, by Proposition 3.1, there exist a subsequence (still denoted $\varepsilon$ ) and $\widehat{w}_{j} \in L^{p}(\Omega \times$ $Y)$, with $\frac{\partial \widehat{w}_{j}}{\partial y_{i}} \in L^{p}(\Omega \times Y)$ such that, for $i \neq j$,

$$
\begin{aligned}
& \mathcal{T}_{\varepsilon}\left(\frac{\partial \mathcal{Q}_{\varepsilon}\left(w_{\varepsilon}\right)}{\partial x_{j}}\right) \rightarrow \widehat{w}_{j} \quad \text { weakly in } L^{p}(\Omega \times Y), \\
& \varepsilon \mathcal{T}_{\varepsilon}\left(\frac{\partial^{2} \mathcal{Q}_{\varepsilon}\left(w_{\varepsilon}\right)}{\partial x_{i} \partial x_{j}}\right) \rightarrow \frac{\partial \widehat{w}_{j}}{\partial y_{i}} \quad \text { weakly in } L^{p}(\Omega \times Y),
\end{aligned}
$$


where $\widehat{w}_{j}$ is $y_{i}$-periodic for every $i \neq j$. Moreover, from Remark 4.6, the function $\widehat{w}_{j}$ does not depend on $y_{j}$, hence it is $Y$-periodic. But, by Remark 4.6 again, $\widehat{w}_{j}$ is also piecewise linear, with respect to any variable $y_{i}$. Consequently, $\widehat{w}_{j}$ is independent of $y$. On the other hand, from (ii) above we have

$$
\frac{\partial \mathcal{Q}_{\varepsilon}\left(w_{\varepsilon}\right)}{\partial x_{j}} \rightarrow \frac{\partial w}{\partial x_{j}} \quad \text { weakly in } L^{p}(\Omega) .
$$

Now Proposition 2.9(iii) gives $\widehat{w}_{j}=\frac{\partial w}{\partial x_{j}}$, and convergence (iii) holds for the whole sequence $\varepsilon$.

Proposition 4.8 (Theorem 3.5 revisited). Let $\left\{w_{\varepsilon}\right\}$ be a sequence converging weakly in $W_{0}^{1, p}(\Omega)$ (resp. in $W^{1, p}(\Omega)$ ) to $w$. Then, up to a subsequence there exists some $\widehat{w}^{\prime}$ in the space $L^{p}\left(\Omega ; W_{\text {per }}^{1, p}(Y)\right)$ such that the following convergences hold:

$$
\begin{aligned}
& \frac{1}{\varepsilon} \mathcal{T}_{\varepsilon}\left(\mathcal{R}_{\varepsilon}\left(w_{\varepsilon}\right)\right) \rightarrow \widehat{w}^{\prime} \quad \text { weakly in } L^{p}\left(\Omega ; W^{1, p}(Y)\right), \\
& \mathcal{T}_{\varepsilon}\left(\nabla \mathcal{R}_{\varepsilon}\left(w_{\varepsilon}\right)\right) \rightarrow \nabla_{y} \widehat{w}^{\prime} \quad \text { weakly in } L^{p}(\Omega \times Y), \\
& \mathcal{T}_{\varepsilon}\left(\nabla w_{\varepsilon}\right) \rightarrow \nabla w+\nabla_{y} \widehat{w}^{\prime} \quad \text { weakly in } L^{p}(\Omega \times Y) .
\end{aligned}
$$

Actually, the connection with the $\widehat{w}$ of Theorem 3.5 is given by

$$
\widehat{w}=\widehat{w}^{\prime}-\mathcal{M}_{Y}\left(\widehat{w}^{\prime}\right)
$$

Proof. Due to estimates of Proposition 4.5, up to a subsequence, there exists $\widehat{w}^{\prime}$ in $L^{p}\left(\Omega ; W_{p e r}^{1, p}(Y)\right)$ such that

$$
\begin{aligned}
& \frac{1}{\varepsilon} \mathcal{T}_{\varepsilon}\left(\mathcal{R}_{\varepsilon}\left(w_{\varepsilon}\right)\right) \rightarrow \widehat{w}^{\prime} \quad \text { weakly in } L^{p}\left(\Omega ; W^{1, p}(Y)\right), \\
& \mathcal{T}_{\varepsilon}\left(\nabla \mathcal{R}_{\varepsilon}\left(w_{\varepsilon}\right)\right) \rightarrow \nabla_{y} \widehat{w}^{\prime} \quad \text { weakly in } L^{p}(\Omega \times Y) .
\end{aligned}
$$

Combining with convergence (iii) of Proposition 4.7 shows that

$$
\mathcal{T}_{\varepsilon}\left(\nabla w_{\varepsilon}\right) \rightarrow \nabla w+\nabla_{y} \widehat{w}^{\prime} \quad \text { weakly in } L^{p}(\Omega \times Y) .
$$

So $\nabla_{y} \widehat{w} \equiv \nabla_{y} \widehat{w}^{\prime}$ in $L^{p}(\Omega \times Y)$. Since $\mathcal{M}_{Y}(\widehat{w})=0$, it follows that $\widehat{w}=\widehat{w}^{\prime}-$ $\mathcal{M}_{Y}\left(\widehat{w}^{\prime}\right)$.

Remark 4.9. In the previous proposition, one can actually compute the average of $\widehat{w}^{\prime}$. One can check that $\mathcal{M}_{Y}\left(\widehat{w}^{\prime}\right)=-\mathcal{M}_{Y}(y) \cdot \nabla w$, and consequently,

$$
\frac{1}{\varepsilon}\left(\mathcal{T}_{\varepsilon}\left(w_{\varepsilon}\right)-\mathcal{M}_{\varepsilon}\left(w_{\varepsilon}\right)\right) \rightarrow y \cdot \nabla w+\widehat{w}^{\prime} \quad \text { weakly in } L^{p}\left(\Omega ; W^{1, p}(Y)\right)
$$

\section{Periodic unfolding and the standard homogenization problem.}

Definition 5.1. Let $\alpha, \beta \in \mathbb{R}$, such that $0<\alpha<\beta$ and $\mathcal{O}$ be an open subset of $\mathbb{R}^{n}$. Denote by $M(\alpha, \beta, \mathcal{O})$ the set of the $n \times n$ matrices $A=\left(a_{i j}\right)_{1 \leq i, j \leq n} \in$ $\left(L^{\infty}(\mathcal{O})\right)^{n \times n}$ such that, for any $\lambda \in \mathbb{R}^{n}$ and a.e. on $\mathcal{O}$,

$$
(A(x) \lambda, \lambda) \geq \alpha|\lambda|^{2}, \quad|A(x) \lambda| \leq \beta|\lambda| .
$$


Let $A^{\varepsilon}=\left(a_{i j}^{\varepsilon}\right)_{1 \leq i, j \leq n}$ be a sequence of matrices in $M(\alpha, \beta, \Omega)$. For $f$ given in $H^{-1}(\Omega)$, consider the Dirichlet problem

$$
\left\{\begin{array}{c}
-\operatorname{div}\left(A^{\varepsilon} \nabla u_{\varepsilon}\right)=f \quad \text { in } \Omega, \\
u^{\varepsilon}=0 \quad \text { on } \partial \Omega .
\end{array}\right.
$$

By the Lax-Milgram theorem, there exists a unique $u^{\varepsilon} \in H_{0}^{1}(\Omega)$ satisfying

$$
\int_{\Omega} A^{\varepsilon} \nabla u_{\varepsilon} \nabla v d x=\langle f, v\rangle_{H^{-1}(\Omega), H_{0}^{1}(\Omega)}, \quad \forall v \in H_{0}^{1}(\Omega),
$$

which is the variational formulation of (5.1). Moreover, one has the apriori estimate

$$
\left\|u_{\varepsilon}\right\|_{H_{0}^{1}(\Omega)} \leq \frac{1}{\alpha}\|f\|_{H^{-1}(\Omega)} .
$$

Consequently, there exist $u_{0}$ in $H_{0}^{1}(\Omega)$ and a subsequence, still denoted $\varepsilon$, such that

$$
u_{\varepsilon} \rightarrow u_{0} \quad \text { weakly in } H_{0}^{1}(\Omega) .
$$

We are now interested to give a limit problem, the "homogenized" problem, satisfied by $u_{0}$. This is called standard homogenization, and the answer, for some classes of $A^{\varepsilon}$, can be found in many works, starting with the classical book by Bensoussan, Lions, and Papanicolaou [11] (see, for instance, Cioranescu and Donato [30] and the references herein). We now recall it.

TheOREM 5.2 (standard periodic homogenization). Let $A=\left(a_{i j}\right)_{1 \leq i, j \leq n}$ belong to $M(\alpha, \beta, Y)$, where $a_{i j}=a_{i j}(y)$ are $Y$-periodic. Set

$$
A^{\varepsilon}(x)=\left(a_{i j}\left(\frac{x}{\varepsilon}\right)\right)_{1 \leq i, j \leq n} \quad \text { a.e. on } \Omega .
$$

Let $u_{\varepsilon}$ be the solution of the corresponding problem (5.1), with $f$ in $H^{-1}(\Omega)$. Then the whole sequence $\left\{u_{\varepsilon}\right\}$ converges to a limit $u_{0}$, which is the unique solution of the homogenized problem

$$
\left\{\begin{array}{l}
-\operatorname{div}\left(A^{0} \nabla u_{0}\right)=\sum_{i, j=1}^{n} a_{i j}^{0} \frac{\partial^{2} u_{0}}{\partial x_{i} \partial x_{j}}=f \quad \text { in } \Omega, \\
u_{0}=0 \quad \text { on } \partial \Omega,
\end{array}\right.
$$

where the constant matrix $A^{0}=\left(a_{i j}^{0}\right)_{1 \leq i, j \leq n}$ is elliptic and given by

$$
a_{i j}^{0}=\mathcal{M}_{Y}\left(a_{i j}-\sum_{k=1}^{n} a_{i k} \frac{\partial \widehat{\chi}_{j}}{\partial y_{k}}\right)=\mathcal{M}_{Y}\left(a_{i j}\right)-\mathcal{M}_{Y}\left(\sum_{k=1}^{n} a_{i k} \frac{\partial \widehat{\chi}_{j}}{\partial y_{k}}\right) .
$$

In (5.7), the functions $\widehat{\chi}_{j}(j=1, \ldots, n)$, often referred to as correctors, are the solutions of the cell systems

$$
\left\{\begin{array}{l}
-\sum_{i, k=1}^{n} \frac{\partial}{\partial y_{i}}\left(a_{i k} \frac{\partial\left(\widehat{\chi}_{j}-y_{j}\right)}{\partial y_{k}}\right)=0 \quad \text { in } Y \\
\mathcal{M}_{Y}\left(\widehat{\chi}_{j}\right)=0 \\
\widehat{\chi}_{j} \quad Y \text {-periodic. }
\end{array}\right.
$$


As will be seen below, using the periodic unfolding, the proof of this theorem is elementary! Actually, with the same proof, a more general result can be obtained, with matrices $A^{\varepsilon}$.

THEOREM 5.3 (periodic homogenization via unfolding). Let $u_{\varepsilon}$ be the solution of problem (5.1), with $f$ in $H^{-1}(\Omega)$, and $A^{\varepsilon}=\left(a_{i j}^{\varepsilon}\right)_{1 \leq i, j \leq n}$ be a sequence of matrices in $M(\alpha, \beta, \Omega)$. Suppose that there exists a matrix $B$ such that

$$
B^{\varepsilon} \doteq \mathcal{T}_{\varepsilon}\left(A^{\varepsilon}\right) \rightarrow B \quad \text { strongly in }\left[L^{1}(\Omega \times Y)\right]^{n \times n} .
$$

Then there exists $u_{0} \in H_{0}^{1}(\Omega)$ and $\widehat{u} \in L^{2}\left(\Omega ; H_{p e r}^{1}(Y)\right)$ such that

$$
\begin{aligned}
& u_{\varepsilon} \rightarrow u_{0} \quad \text { weakly in } H_{0}^{1}(\Omega), \\
& \mathcal{T}_{\varepsilon}\left(u_{\varepsilon}\right) \rightarrow u_{0} \quad \text { weakly in } L^{2}\left(\Omega ; H^{1}(Y)\right), \\
& \mathcal{T}_{\varepsilon}\left(\nabla u_{\varepsilon}\right) \rightarrow \nabla u_{0}+\nabla_{y} \widehat{u} \quad \text { weakly in } L^{2}(\Omega \times Y),
\end{aligned}
$$

and the pair $\left(u_{0}, \widehat{u}\right)$ is the unique solution of the problem

$$
\left\{\begin{aligned}
& \forall \Psi \in H_{0}^{1}(\Omega), \forall \Phi \in L^{2}\left(\Omega ; H_{p e r}^{1}(Y)\right) \\
& \frac{1}{|Y|} \int_{\Omega \times Y} B(x, y)\left[\nabla u_{0}(x)\right.\left.+\nabla_{y} \widehat{u}(x, y)\right]\left[\nabla \Psi(x)+\nabla_{y} \Phi(x, y)\right] d x d y \\
&=\langle f, \Psi\rangle_{H^{-1}(\Omega), H_{0}^{1}(\Omega)} .
\end{aligned}\right.
$$

Remark 5.4. System (5.11) is the unfolded formulation of the homogenized limit problem. It is of standard variational form in the space

$$
\mathcal{H}=H_{0}^{1}(\Omega) \times L^{2}\left(\Omega ; H_{\text {per }}^{1}(Y) / \mathbb{R}\right) .
$$

Remark 5.5. Hypothesis (5.9) implies that $B \in M(\alpha, \beta, \Omega \times Y)$.

Remark 5.6. If $A^{\varepsilon}$ is of the form (5.5), then $B(x, y)=A(y)$. In the case where $A^{\varepsilon}(x)=A_{1}(x) A_{2}\left(\frac{x}{\varepsilon}\right)$, one has (5.9), with $B(x, y)=A_{1}(x) A_{2}(y)$.

Remark 5.7. Let us point out that every matrix $B \in M(\alpha, \beta, \Omega \times Y)$ can be approached by the sequence of matrices $A^{\varepsilon}$ in $M(\alpha, \beta, \Omega)$, with $A^{\varepsilon}$ defined as follows:

$$
A^{\varepsilon}= \begin{cases}\mathcal{U}_{\varepsilon}(B) & \text { in } \widehat{\Omega}_{\varepsilon}, \\ \alpha I_{n} & \text { in } \Lambda_{\varepsilon} .\end{cases}
$$

Proof of Theorem 5.3. Convergences (5.10) follow from estimate (5.3), Corollary 3.3, and Proposition 4.7, respectively.

Let us choose $v=\Psi$, with $\Psi \in H_{0}^{1}(\Omega)$ as test function in (5.2). The integration formula (2.5) from Proposition 2.7 gives

$$
\frac{1}{|Y|} \int_{\Omega \times Y} B^{\varepsilon} \mathcal{T}_{\varepsilon}\left(\nabla u_{\varepsilon}\right) \mathcal{T}_{\varepsilon}(\nabla \Psi) d x d y \stackrel{\mathcal{I}_{\varepsilon}}{\simeq}\langle f, \Psi\rangle_{H^{-1}(\Omega), H_{0}^{1}(\Omega)} .
$$

We are allowed to pass to the limit in (5.12), due to (5.9), (5.10), and Proposition 2.9(i), to get

$$
\frac{1}{|Y|} \int_{\Omega \times Y} B(x, y)\left[\nabla u_{0}(x)+\nabla_{y} \widehat{u}(x, y)\right] \nabla \Psi(x) d x d y=\langle f, \Psi\rangle_{H^{-1}(\Omega), H_{0}^{1}(\Omega)} .
$$


Now, taking in (5.2), as test function $v^{\varepsilon}(x)=\varepsilon \Psi(x) \psi\left(\frac{x}{\varepsilon}\right), \Psi \in \mathcal{D}(\Omega), \psi \in H_{\text {per }}^{1}(Y)$, one has, due to (2.5) and Proposition 2.7,

$$
\begin{aligned}
& \frac{1}{|Y|} \int_{\Omega \times Y} B^{\varepsilon} \mathcal{T}_{\varepsilon}\left(\nabla u_{\varepsilon}\right) \varepsilon \psi(y) \mathcal{T}_{\varepsilon}(\nabla \Psi) d x d y \\
& \quad+\frac{1}{|Y|} \int_{\Omega \times Y} B^{\varepsilon} \mathcal{T}_{\varepsilon}\left(\nabla u_{\varepsilon}\right) \nabla_{y} \psi(y) \mathcal{T}_{\varepsilon}(\Psi) d x d y \stackrel{\mathcal{T}_{\varepsilon}}{\simeq}\left\langle f, v_{\varepsilon}\right\rangle_{H^{-1}(\Omega), H_{0}^{1}(\Omega)} .
\end{aligned}
$$

Since $v^{\varepsilon} \rightarrow 0$ in $H_{0}^{1}(\Omega)$, we get at the limit

$$
\frac{1}{|Y|} \int_{\Omega \times Y} B(x, y)\left[\nabla u_{0}(x)+\nabla_{y} \widehat{u}(x, y)\right] \Psi(x) \nabla_{y} \psi(y) d x d y=0 .
$$

By the density of the tensor product $\mathcal{D}(\Omega) \otimes H_{p e r}^{1}(Y)$ in $L^{2}\left(\Omega ; H_{p e r}^{1}(Y)\right)$, this holds for all $\Phi$ in $L^{2}\left(\Omega ; H_{p e r}^{1}(Y)\right)$.

Remark 5.8. As in the two-scale method, (5.11) gives $\widehat{u}$ in terms of $\nabla u_{0}$ and yields the standard form of the homogenized equation, i.e., (5.6). In the simple case where $A(x, y)=A(y)=\left(a_{i j}(y)\right)_{1 \leq i, j \leq n}$, it is easily seen that

$$
\widehat{u}=\sum_{i=1}^{n} \frac{\partial u_{0}}{\partial x_{i}} \widehat{\chi}_{i},
$$

and that the limit $B$ is precisely the matrix $A^{0}$ which was defined in Theorem 5.2 by (5.7) and (5.8).

Proposition 5.9 (convergence of the energy). Under the hypotheses of Theorem 5.3,

$$
\left\{\begin{array}{l}
\lim _{\varepsilon \rightarrow 0} \int_{\Omega} A^{\varepsilon} \nabla u_{\varepsilon} \nabla u_{\varepsilon} d x=\frac{1}{|Y|} \int_{\Omega \times Y} B\left[\nabla u_{0}+\nabla_{y} \widehat{u}\right]\left[\nabla u_{0}+\nabla_{y} \widehat{u}\right] d x d y, \\
\lim _{\varepsilon \rightarrow 0} \int_{\Lambda_{\varepsilon}}\left|\nabla u_{\varepsilon}\right|^{2} d x=0 .
\end{array}\right.
$$

Proof. By standard weak lower-semicontinuity, one successively obtains

$$
\begin{aligned}
\frac{1}{|Y|} \int_{\Omega \times Y} B\left[\nabla u_{0}\right. & \left.+\nabla_{y} \widehat{u}\right]\left[\nabla u_{0}+\nabla_{y} \widehat{u}\right] d x d y \\
& \leq \liminf _{\varepsilon \rightarrow 0} \frac{1}{|Y|} \int_{\Omega \times Y} B^{\varepsilon} \mathcal{T}_{\varepsilon}\left(\nabla u_{\varepsilon}\right) \mathcal{T}_{\varepsilon}\left(\nabla u_{\varepsilon}\right) d x d y \\
& \leq \limsup _{\varepsilon \rightarrow 0} \frac{1}{|Y|} \int_{\Omega \times Y} B^{\varepsilon} \mathcal{T}_{\varepsilon}\left(\nabla u_{\varepsilon}\right) \mathcal{T}_{\varepsilon}\left(\nabla u_{\varepsilon}\right) d x d y \\
& \leq \limsup _{\varepsilon \rightarrow 0} \int_{\Omega} A^{\varepsilon} \nabla u_{\varepsilon} \nabla u_{\varepsilon} d x=\limsup _{\varepsilon \rightarrow 0}\left\langle f, u_{\varepsilon}\right\rangle_{H^{-1}(\Omega), H_{0}^{1}(\Omega)} \\
& =\left\langle f, u_{0}\right\rangle_{H^{-1}(\Omega), H_{0}^{1}(\Omega)}=\frac{1}{|Y|} \int_{\Omega \times Y} B\left[\nabla u_{0}+\nabla_{y} \widehat{u}\right]\left[\nabla u_{0}+\nabla_{y} \widehat{u}\right] d x d y,
\end{aligned}
$$

which gives the first convergence in (5.15), as well as

$$
\limsup _{\varepsilon \rightarrow 0} \int_{\Lambda_{\varepsilon}} A^{\varepsilon} \nabla u_{\varepsilon} \nabla u_{\varepsilon} d x=0,
$$

which implies the second convergence in (5.15). 
Remark 5.10. From the above proof, we also have

$$
\begin{aligned}
\lim _{\varepsilon \rightarrow 0} \frac{1}{|Y|} \int_{\Omega \times Y} B^{\varepsilon} \mathcal{T}_{\varepsilon}\left(\nabla u_{\varepsilon}\right) \mathcal{T}_{\varepsilon}\left(\nabla u_{\varepsilon}\right) d x d y & \\
& =\frac{1}{|Y|} \int_{\Omega \times Y} B\left[\nabla u_{0}+\nabla_{y} \widehat{u}\right]\left[\nabla u_{0}+\nabla_{y} \widehat{u}\right] d x d y .
\end{aligned}
$$

COROLlaRY 5.11. The following strong convergence holds:

$$
\mathcal{T}_{\varepsilon}\left(\nabla u_{\varepsilon}\right) \rightarrow \nabla u_{0}+\nabla_{y} \widehat{u} \quad \text { strongly in } L^{2}(\Omega \times Y) .
$$

Proof. We have successively

$$
\begin{aligned}
\frac{1}{|Y|} \int_{\Omega \times Y} B^{\varepsilon}\left[\mathcal{T}_{\varepsilon}\left(\nabla u_{\varepsilon}\right)\right. & \left.-\nabla u_{0}-\nabla_{y} \widehat{u}\right]\left[\mathcal{T}_{\varepsilon}\left(\nabla u_{\varepsilon}\right)-\nabla u_{0}-\nabla_{y} \widehat{u}\right] d x d y \\
& =\frac{1}{|Y|} \int_{\Omega \times Y} B^{\varepsilon} \mathcal{T}_{\varepsilon}\left(\nabla u_{\varepsilon}\right) \mathcal{T}_{\varepsilon}\left(\nabla u_{\varepsilon}\right) d x d y \\
& -\frac{1}{|Y|} \int_{\Omega \times Y} B^{\varepsilon}\left[\nabla u_{0}+\nabla_{y} \widehat{u}\right] \mathcal{T}_{\varepsilon}\left(\nabla u_{\varepsilon}\right) d x d y \\
& -\frac{1}{|Y|} \int_{\Omega \times Y} B^{\varepsilon} \mathcal{T}_{\varepsilon}\left(\nabla u_{\varepsilon}\right)\left[\nabla u_{0}+\nabla_{y} \widehat{u}\right] d x d y \\
& +\frac{1}{|Y|} \int_{\Omega \times Y} B^{\varepsilon}\left[\nabla u_{0}+\nabla_{y} \widehat{u}\right]\left[\nabla u_{0}+\nabla_{y} \widehat{u}\right] d x d y .
\end{aligned}
$$

Each term in the right-hand side converges, the first one due to Remark 5.10, and the others due to (5.10) and hypothesis (5.9). So, the right-hand side term converges to zero. Then convergence (5.16) is a consequence of the ellipticity of $B^{\varepsilon}$.

Remark 5.12. One can consider problem (5.1) with a homogeneous Neumann boundary condition on $\partial \Omega$ provided a zero order term is added to the operator. This problem is variational on the space $H^{1}(\Omega)$ without any regularity condition on the boundary. The exact same method applies and gives the corresponding limit problem. In order for a nonhomogeneous Neumann boundary condition (or Robin condition) on $\partial \Omega$ to make sense, a well-behaved trace operator is needed from $H^{1}(\Omega)$ to $L^{2}(\Omega)$. In that case, the same method applies.

6. Some corrector results and error estimates. Under additional regularity assumptions on the homogenized solution $u_{0}$ and the cell-functions $\widehat{\chi}_{j}$, the strong convergence for the gradient of $u_{0}$ with a corrector is known (cf. [11] Chapter 1, section 5, [30] Chapter 8, section 3 and references therein). More precisely, suppose that $\nabla_{y} \widehat{\chi}_{j} \in\left(L^{r}(Y)\right)^{n}, j=1, \ldots, n$ and $\nabla u^{0} \in L^{s}(\Omega)$, with $1 \leq r, s<+\infty$ and such that $1 / r+1 / s=1 / 2$. Then

$$
\nabla u_{\varepsilon}-\nabla u_{0}-\sum_{j=1}^{n} \frac{\partial u_{0}}{\partial x_{j}}\left(\nabla_{y} \widehat{\chi}_{j}\right)\left(\frac{\dot{q}}{\varepsilon}\right) \rightarrow 0 \quad \text { strongly in } L^{2}(\Omega) .
$$

Our next result gives a corrector result without any additional regularity assumption on $\hat{\chi}_{j}$, and its proof reduces to a few lines. We also include a new type of corrector.

TheOREM 6.1. Under the hypotheses of Theorem 5.2, one has

$$
\nabla u_{\varepsilon}-\nabla u_{0}-\mathcal{U}_{\varepsilon}\left(\nabla_{y} \widehat{u}\right) \rightarrow 0 \quad \text { strongly in } L^{2}(\Omega) \text {. }
$$


In the case where $A^{\varepsilon}(x)=A\left(\left\{\frac{x}{\varepsilon}\right\}_{Y}\right)$, the function $u_{0}+\varepsilon \sum_{i=1}^{n} \mathcal{Q}_{\varepsilon}\left(\frac{\partial u_{0}}{\partial x_{i}}\right) \chi_{i}\left(\{\dot{\bar{\varepsilon}}\}_{Y}\right)$ belongs to $H^{1}(\Omega)$, and one has

$$
u_{\varepsilon}-u_{0}-\varepsilon \sum_{i=1}^{n} \mathcal{Q}_{\varepsilon}\left(\frac{\partial u_{0}}{\partial x_{i}}\right) \chi_{i}\left(\{\dot{\dot{\varepsilon}}\}_{Y}\right) \rightarrow 0 \quad \text { strongly in } H^{1}(\Omega) .
$$

Proof. From (5.15), (5.16), and Proposition 2.18(iii), one immediately has

$$
\nabla u_{\varepsilon}-\mathcal{U}_{\varepsilon}\left(\nabla u_{0}\right)-\mathcal{U}_{\varepsilon}\left(\nabla_{y} \widehat{u}\right) \rightarrow 0 \quad \text { strongly in } L^{2}(\Omega) .
$$

But since $\nabla u_{0}$ belongs to $L^{2}(\Omega)$, Corollary 2.26 implies that

$$
\mathcal{U}_{\varepsilon}\left(\nabla u_{0}\right) \rightarrow \nabla u_{0} \quad \text { strongly in } L^{2}(\Omega),
$$

whence (6.1). From (4.4) in Proposition 4.2, the function $u_{0}+\varepsilon \sum_{i=1}^{n} \mathcal{Q}_{\varepsilon}\left(\frac{\partial u_{0}}{\partial x_{i}}\right) \chi_{i}\left(\{\dot{\bar{\varepsilon}}\}_{Y}\right)$ belongs to $H^{1}(\Omega)$. From (5.14), we obtain

$$
\begin{aligned}
\nabla u_{0}+\mathcal{U}_{\varepsilon}\left(\nabla_{y} \widehat{u}\right) & -\nabla\left[u_{0}+\varepsilon \sum_{i=1}^{n} \mathcal{Q}_{\varepsilon}\left(\frac{\partial u_{0}}{\partial x_{i}}\right) \chi_{i}\left(\left\{\frac{\dot{\varepsilon}}{\varepsilon}\right\}_{Y}\right)\right] \\
= & -\sum_{i=1}^{n}\left[\mathcal{Q}_{\varepsilon}\left(\frac{\partial u_{0}}{\partial x_{i}}\right)-\mathcal{M}_{\varepsilon}\left(\frac{\partial u_{0}}{\partial x_{i}}\right)\right] \nabla_{y} \chi_{i}\left(\left\{\dot{\frac{1}{\varepsilon}}\right\}_{Y}\right) \\
& -\varepsilon \sum_{i=1}^{n} \nabla\left[\mathcal{Q}_{\varepsilon}\left(\frac{\partial u_{0}}{\partial x_{i}}\right)\right] \chi_{i}\left(\left\{\frac{\dot{\varepsilon}}{\varepsilon}\right\}_{Y}\right),
\end{aligned}
$$

and using estimate (4.2), Proposition 2.25(iii), and Corollary 4.3, one immediately gets the strong convergence in $L^{2}(\Omega)$ of the right-hand side in the above equality. Thanks to (6.1), one has (6.2).

We end this section by recalling the error estimates obtained by Griso in [42], [44], and [45] for problem (5.1), with $f \in L^{2}(\Omega)$.

TheOREM 6.2 (see [42], [44]). Suppose that $\partial \Omega$ is of class $\mathcal{C}^{1,1}$. The solution $u_{\varepsilon}$ of (5.1) satisfies the following estimates:

$$
\begin{aligned}
& \left\|\nabla u_{\varepsilon}-\nabla u_{0}-\sum_{i=1}^{n} \mathcal{Q}_{\varepsilon}\left(\frac{\partial u_{0}}{\partial x_{i}}\right) \nabla_{y} \widehat{\chi}_{i}\left(\left\{\frac{\dot{\varepsilon}}{\varepsilon}\right\}\right)\right\|_{\left[L^{2}(\Omega)\right]^{n}} \leq C \varepsilon^{1 / 2}\|f\|_{L^{2}(\Omega)}, \\
& \left\|u_{\varepsilon}-u_{0}\right\|_{L^{2}(\Omega)}+\left\|\rho\left(\nabla u_{\varepsilon}-\nabla u_{0}-\sum_{i=1}^{n} \mathcal{Q}_{\varepsilon}\left(\frac{\partial u_{0}}{\partial x_{i}}\right) \nabla_{y} \widehat{\chi}_{i}(\dot{\bar{\varepsilon}})\right)\right\|_{\left[L^{2}(\Omega)\right]^{n}} \leq C \varepsilon\|f\|_{L^{2}(\Omega)},
\end{aligned}
$$

where $\hat{\chi}_{i}$ for $i=1, \ldots, n$ is defined by (5.8) and $\rho=\rho(x)$ is the distance between $x \in \Omega$ and the boundary $\partial \Omega$. The constant $C$ depends on $n, A$, and $\partial \Omega$.

COROLlary 6.3 (see [44]). Let $\Omega^{\prime}$ be an open set strongly included in $\Omega$, then

$$
\left\|u_{\varepsilon}-u_{0}-\varepsilon \sum_{i=1}^{n} \mathcal{Q}_{\varepsilon}\left(\frac{\partial u_{0}}{\partial x_{i}}\right) \widehat{\chi}_{i}\left(\frac{\dot{\varepsilon}}{\varepsilon}\right)\right\|_{H^{1}\left(\Omega^{\prime}\right)} \leq C \varepsilon\|f\|_{L^{2}(\Omega)} .
$$

The constant depends on $n, A, \Omega^{\prime}$, and $\partial \Omega$.

In what follows in this paragraph, we suppose that the open set $\Omega$ is a bounded domain in $\mathbb{R}^{n}, n=2$ or 3 , of polygonal $(n=2)$ or polyhedral $(n=3)$ boundary. We 
assume that $\Omega$ is on one side only of its boundary, and that $\Gamma_{0}$ is the union of some edges $(n=2)$ or some faces $(n=3)$ of $\partial \Omega$. Recall that classical regularity results show that the solution $u_{0}$ of the homogenized problem $(5.6)$ belongs to $H^{1+s}(\Omega)$ for $s$ in $] 1 / 2,1$ [ ( $s=1$ if the domain is convex $)$ depending only on $\partial \Omega$, on $A^{0}$, and satisfies the estimate

$$
\left\|\nabla u_{0}\right\|_{H^{1+s}(\Omega)} \leq C\|f\|_{L^{2}(\Omega)} .
$$

The error estimate for this case is given in the following result.

THEOREM 6.4 (see [45]). The solution $u_{\varepsilon}$ of problem (5.1) satisfies the estimate

$$
\begin{aligned}
& \left\|\nabla u_{\varepsilon}-\nabla u_{0}-\sum_{i=1}^{n} \mathcal{Q}_{\varepsilon}\left(\frac{\partial u_{0}}{\partial x_{i}}\right) \nabla_{y} \widehat{\chi}_{i}\left(\frac{\dot{q}}{\varepsilon}\right)\right\|_{\left[L^{2}(\Omega)\right]^{n}} \leq C \varepsilon^{s / 2}\|f\|_{L^{2}(\Omega)}, \\
& \left\|u_{\varepsilon}-u_{0}\right\|_{L^{2}(\Omega)}+\left\|\rho\left(\nabla u_{\varepsilon}-\nabla u_{0}-\sum_{i=1}^{n} \mathcal{Q}_{\varepsilon}\left(\frac{\partial u_{0}}{\partial x_{i}}\right) \nabla_{y} \widehat{\chi}_{i}\left(\frac{\dot{ }}{\varepsilon}\right)\right)\right\|_{\left[L^{2}(\Omega)\right]^{n}} \leq C \varepsilon^{s}\|f\|_{L^{2}(\Omega)} .
\end{aligned}
$$

The constants depend on $n, A$, and $\partial \Omega$.

COROLlary 6.5 (see [45]). Let $\Omega^{\prime}$ be an open set strongly included in $\Omega$, then

$$
\left\|u_{\varepsilon}-u_{0}-\varepsilon \sum_{i=1}^{n} \mathcal{Q}_{\varepsilon}\left(\frac{\partial u_{0}}{\partial x_{i}}\right) \widehat{\chi}_{i}\left(\frac{\dot{\varepsilon}}{\varepsilon}\right)\right\|_{H^{1}\left(\Omega^{\prime}\right)} \leq C \varepsilon^{s}\|f\|_{L^{2}(\Omega)} .
$$

The constant depends on $n, A, \Omega^{\prime}$, and $\partial \Omega$.

7. Periodic unfolding and multiscales. As we mentioned in the Introduction, the periodic unfolding method turns out to be particularly well-adapted to multiscales problems. As an example, we treat here a problem with two different small scales.

Consider two periodicity cells $Y$ and $Z$, both having the properties introduced at the beginning of section 2 (each associated to its set of periods). Suppose that $Y$ is "partitioned" in two nonempty disjoint open subsets $Y_{1}$ and $Y_{2}$, i.e., such that $Y_{1} \cap Y_{2}=\emptyset$ and $\bar{Y}=\bar{Y}_{1} \cup \bar{Y}_{2}$.

Let $A^{\varepsilon \delta}$ be a matrix field defined by

$$
A^{\varepsilon \delta}(x)= \begin{cases}A_{1}\left(\left\{\frac{x}{\varepsilon}\right\}_{Y}\right) & \text { for } \quad\left\{\frac{x}{\varepsilon}\right\}_{Y} \in Y_{1}, \\ A_{2}\left(\left\{\frac{\left\{\frac{x}{\varepsilon}\right\}_{Y}}{\delta}\right\}_{Z}\right) & \text { for }\left\{\frac{x}{\varepsilon}\right\}_{Y} \in Y_{2},\end{cases}
$$

where $A_{1}$ is in $M\left(\alpha, \beta, Y_{1}\right)$ and $A_{2}$ in $M(\alpha, \beta, Z)$ (cf. Definition 5.1). Here we have two small scales, namely, $\varepsilon$ and $\varepsilon \delta$, associated, respectively, to the cells $Y$ and $Z$ (see Figure 7).

Consider the problem

$$
\int_{\Omega} A^{\varepsilon \delta} \nabla u_{\varepsilon \delta} \nabla w d x=\int_{\Omega} f w d x \quad \forall w \in H_{0}^{1}(\Omega),
$$

with $f$ in $L^{2}(\Omega)$. The Lax-Milgram theorem immediately gives the existence and uniqueness of $u_{\varepsilon \delta}$ in $H_{0}^{1}(\Omega)$ satisfying the estimate

$$
\left\|u_{\varepsilon \delta}\right\|_{H_{0}^{1}(\Omega)} \leq \frac{1}{\alpha}\|f\|_{L^{2}(\Omega)} .
$$




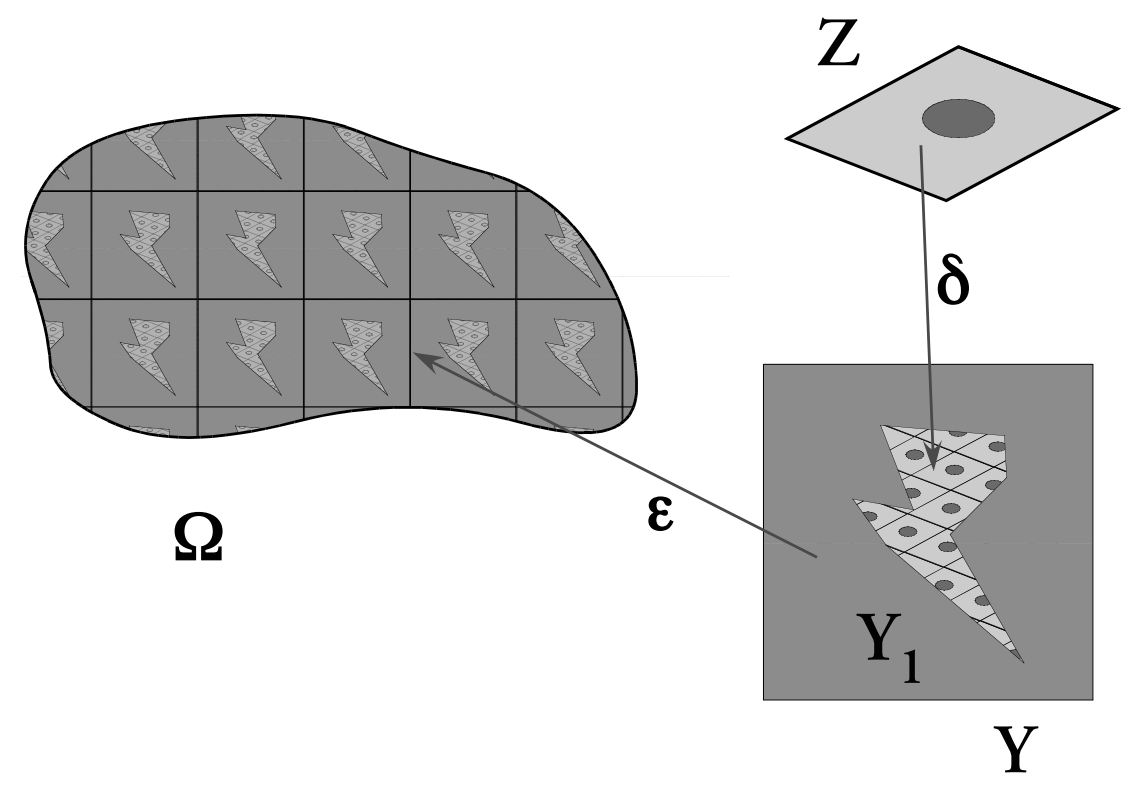

FIG. 7. A domain with periodic scales $\varepsilon$ and $\varepsilon \delta$.

So, there is some $u_{0}$ such that, up to a subsequence,

$$
u_{\varepsilon \delta} \rightarrow u_{0} \quad \text { weakly in } H_{0}^{1}(\Omega) .
$$

Using the unfolding method for scale $\varepsilon$, as before we have

$$
\begin{aligned}
& \mathcal{Q}_{\varepsilon}\left(u_{\varepsilon \delta}\right) \rightarrow u_{0} \quad \text { weakly in } H_{0}^{1}(\Omega), \\
& \mathcal{T}_{\varepsilon}\left(u_{\varepsilon \delta}\right) \rightarrow u_{0} \quad \text { weakly in } L^{2}\left(\Omega ; H^{1}(Y)\right), \\
& \frac{1}{\varepsilon} \mathcal{T}_{\varepsilon}\left(\mathcal{R}_{\varepsilon}\left(u_{\varepsilon \delta}\right)\right) \rightarrow \widehat{u} \quad \text { weakly in } L^{2}\left(\Omega ; H^{1}(Y)\right), \\
& \mathcal{T}_{\varepsilon}\left(\nabla u_{\varepsilon \delta}\right) \rightarrow \nabla u_{0}+\nabla_{y} \widehat{u} \quad \text { in } L^{2}(\Omega \times Y) .
\end{aligned}
$$

These convergences do not see the oscillations at the scale $\varepsilon \delta$. In order to capture them, one considers the restrictions to the set $\Omega \times Y_{2}$ defined by

$$
\left.v_{\varepsilon \delta}(x, y) \doteq \frac{1}{\varepsilon} \mathcal{T}_{\varepsilon}\left(\mathcal{R}_{\varepsilon}\left(u_{\varepsilon \delta}\right)\right)\right|_{Y_{2}} .
$$

Obviously,

$$
\left.v_{\varepsilon \delta} \rightarrow \widehat{u}\right|_{Y_{2}} \quad \text { weakly in } L^{2}\left(\Omega ; H^{1}\left(Y_{2}\right)\right) .
$$

Now, we apply to $v_{\varepsilon \delta}$, a similar unfolding operation, denoted $\mathcal{T}_{\delta}^{y}$, for the variable $y$, thus adding a new variable $z \in Z$.

$$
\mathcal{T}_{\delta}^{y}\left(v_{\varepsilon \delta}\right)(x, y, z)=v_{\varepsilon \delta}\left(x, \delta\left[\frac{y}{\delta}\right]_{Z}+\delta z\right) \quad \text { for } x \in \Omega, y \in Y_{2}, \text { and } z \in Z .
$$

It is essential to remark that all of the estimates and weak convergence properties which were shown for the original unfolding $\mathcal{T}_{\varepsilon}$ still hold for $\mathcal{T}_{\delta}^{y}$, with $x$ being a mere parameter. For example, Proposition 4.7 and Theorem 3.5 adapted to this case imply that

$$
\begin{aligned}
& \mathcal{T}_{\delta}^{y}\left(\nabla_{y} v_{\varepsilon \delta}\right)-\left.\nabla_{y} \widehat{u}\right|_{\Omega_{2}}+\nabla_{z} \widetilde{u} \quad \text { weakly in } L^{2}\left(\Omega \times Y_{2} \times Z\right), \\
& \mathcal{T}_{\delta}^{y}\left(\mathcal{T}_{\varepsilon}\left(\nabla u_{\varepsilon \delta}\right)\right) \rightarrow \nabla u_{0}+\nabla_{y} \widehat{u}+\nabla_{z} \widetilde{u} \quad \text { weakly in } L^{2}\left(\Omega \times Y_{2} \times Z\right) .
\end{aligned}
$$

Under these conditions, the limit functions $u_{0}, \widehat{u}$, and $\widetilde{u}$ are characterized by the following result. 
THEOREM 7.1. The functions

$$
u_{0} \in H_{0}^{1}(\Omega), \quad \widehat{u} \in L^{2}\left(\Omega, H_{p e r}^{1}(Y) / \mathbb{R}\right), \quad \widetilde{u} \in L^{2}\left(\Omega \times \Omega_{2}, H_{p e r}^{1}(Z) / \mathbb{R}\right)
$$

are the unique solutions of the variational problem

$$
\left\{\begin{array}{l}
\frac{1}{|Y||Z|} \int_{\Omega} \int_{Y_{2}} \int_{Z} A_{2}(z)\left\{\nabla u_{0}+\nabla_{y} \widehat{u}+\nabla_{z} \widetilde{u}\right\}\left\{\nabla \Psi+\nabla_{y} \Phi+\nabla_{z} \Theta\right\} d x d y d z \\
\quad+\frac{1}{|Y|} \int_{\Omega} \int_{Y_{1}} A_{1}(y)\left\{\nabla u_{0}+\nabla_{y} \widehat{u}\right\}\left\{\nabla \Psi+\nabla_{y} \Phi\right\} d x d y=\int_{\Omega} f \Psi d x, \\
\forall \Psi \in H_{0}^{1}(\Omega), \quad \forall \Phi \in L^{2}\left(\Omega ; H_{p e r}^{1}(Y) / \mathbb{R}\right), \forall \Theta \in L^{2}\left(\Omega \times \Omega_{2}, H_{p e r}^{1}(Z) / \mathbb{R}\right) .
\end{array}\right.
$$

The proof uses test functions of the form

$$
\Psi(x)+\varepsilon \Psi_{1}(x) \Phi_{1}\left(\frac{x}{\varepsilon}\right)+\varepsilon \delta \Psi_{2}(x) \Phi_{2}\left(\left\{\frac{x}{\varepsilon}\right\}_{Y}\right) \Theta_{2}\left(\frac{1}{\delta}\left\{\frac{x}{\varepsilon}\right\}_{Y}\right),
$$

where $\Psi, \Psi_{1}, \Psi_{2}$ are in $\mathcal{D}(\Omega), \Phi_{1}$ in $H_{p e r}^{1}(Y), \Phi_{2} \in \mathcal{D}\left(Y_{2}\right)$, and $\Theta_{2} \in H_{\text {per }}^{1}(Z)$.

Remark 7.2. The same theorem holds true for a general $A^{\varepsilon \delta}$ under the hypotheses

$$
\begin{aligned}
& \mathcal{T}_{\varepsilon}\left(A^{\varepsilon \delta}\right) 1_{Y_{1}} \rightarrow A_{1} \quad \text { strongly in }\left[L^{1}\left(\Omega \times Y_{1}\right)\right]^{n \times n}, \\
& \mathcal{T}_{\delta}^{y}\left(\mathcal{T}_{\varepsilon}\left(A^{\varepsilon \delta}\right) 1_{Y_{2}}\right) \rightarrow A_{2} \quad \text { strongly in }\left[L^{1}\left(\Omega \times Y_{2} \times Z\right)\right]^{n \times n} .
\end{aligned}
$$

Proposition 5.9 (convergence of the energy) and Corollary 5.11 extend without any difficulty to the multiscale case.

Proposition 7.3. The convergence for the energy holds true:

$$
\begin{aligned}
\lim _{\varepsilon, \delta \rightarrow 0} \int_{\Omega} A^{\varepsilon \delta} \nabla u_{\varepsilon \delta} \nabla u_{\varepsilon \delta} d x \\
=\frac{1}{|Y||Z|} \int_{\Omega} \int_{Y_{2}} \int_{Z} A_{2}(z)\left\{\nabla u_{0}+\nabla_{y} \widehat{u}+\nabla_{z} \widetilde{u}\right\}\left\{\nabla u_{0}+\nabla_{y} \widehat{u}+\nabla_{z} \widetilde{u}\right\} d x d y d z \\
\quad+\frac{1}{|Y|} \int_{\Omega} \int_{Y_{1}} A_{1}(y)\left\{\nabla u_{0}+\nabla_{y} \widehat{u}\right\}\left\{\nabla u_{0}+\nabla_{y} \widehat{u}\right\} d x d y .
\end{aligned}
$$

COROLlaRY 7.4. The following strong convergences hold true:

$$
\begin{aligned}
& \left.\mathcal{T}_{\delta}^{y}\left(\nabla_{y} v_{\varepsilon \delta}\right) \rightarrow \nabla_{y} \widehat{u}\right|_{\Omega_{2}}+\nabla_{z} \widetilde{u} \quad \text { strongly in } L^{2}\left(\Omega \times Y_{2} \times Z\right), \\
& \mathcal{T}_{\delta}^{y}\left(\mathcal{T}_{\varepsilon}\left(\nabla u_{\varepsilon \delta}\right)\right) \rightarrow \nabla u_{0}+\nabla_{y} \widehat{u}+\nabla_{z} \widetilde{u} \quad \text { strongly in } L^{2}\left(\Omega \times Y_{2} \times Z\right) .
\end{aligned}
$$

Remark 7.5. A corrector result, similar to that of Theorem 6.1, can be obtained.

Remark 7.6. Theorem 7.1 can be extended to the case of any finite number of distinct scales by a simple reiteration.

8. Further developments. The unfolding method has some interesting properties which make it suitable for more general situations than that presented here. In problems which are set on a domain $\Omega_{\varepsilon}$ which depends on the parameter $\varepsilon$, it may be difficult to have a good notion of convergence for the sequence of solutions $u_{\varepsilon}$. The traditional way is to extend the solution by 0 outside $\Omega_{\varepsilon}$; however, this precludes the strong convergence of these extended functions in general. For the case of holes of the 
size of order $\varepsilon$ distributed $\varepsilon$-periodically, the unfolded sequence lives on a fixed domain. Similarly, for domains with $\varepsilon$-oscillating boundaries, a partial unfolding yields a function which is defined on a fixed domain.

We conclude by giving a list of publications making use of the unfolding method in several of these directions (both for linear and nonlinear problems).

- Reiterated homogenization: Meunier and Van Schaftingen [56].

- Electro-magnetism: Banks et al. [7], Bossavit, Griso, and Miara [15].

- Homogenization of thin piezoelectric shells: Ghergu et al. [39].

- Homogenization of diffusion deformation media: Griso and Rohan [46].

- Homogenization of the Stokes problem in porous media: Cioranescu, Damlamian, and Griso [25].

- Homogenization in perforated domains with Robin boundary conditions: Cioranescu, Donato and Zaki [31], [32].

- Homogenization in domains with oscillating boundaries: Damlamian and Pettersson [36].

- Homogenization of nonlinear integrals of the calculus of variations: Cioranescu, Damlamian, and De Arcangelis [27], [28], and [29].

- Homogenization of multivalued monotone operators of Leray-Lions type: Damlamian, Meunier, and Van Schaftingen [37].

- Thin junctions in linear elasticity: Blanchard, Gaudiello, and Griso [12], [13], Blanchard and Griso [14].

- Thin domains and free boundary problems arising in lubrication theory: Bayada, Martin, and Vazquez [9], [10].

- Elasticity problems in perforated domains: Griso and Sanchez-Rua [47].

- Neumann sieve and Dirichlet shield problems: Onofrei [60], Cioranescu et al. [26]. This last paper treats the case of domains with $\varepsilon$-periodically distributed "very small" holes (their size being a power of $\varepsilon$ ) on the boundary of which a homogeneous Dirichlet condition is prescribed. This requires the introduction of a rescaled unfolding operator (which originally appeared in the framework of the two-scale convergence in Casado-Díaz [20]).

Aknowledgments. We thank Petru Mironescu and Riccardo De Arcangelis for helpful comments and corrections.

\section{REFERENCES}

[1] G. Allaire, Homogenization and two-scale convergence, SIAM J. Math. Anal., 23 (1992), pp. $1482-1518$.

[2] G. Allaire and M. Briane, Multiscale convergence and reiterated homogenization, Proc. Roy. Soc. Edinburgh Sect. A, 126 (1996), pp. 297-342.

[3] G. Allaire And C. Conca, Bloch wave homogenization and spectral asymptotic analysis, J. Math. Pures Appl., 2 (1998), pp. 153-208.

[4] G. Allaire, C. Conca, And M. Vanninathan, Spectral asymptotics of the Helmholtz model in fluid-solid structures, Internat. J. Numer. Methods Engrg., 9 (1999), pp. 1463-1504.

[5] T. Arbogast, J. Douglas, And U. Hornung, Derivation of the double porosity model of single phase flow via homogenization theory, SIAM J. Math. Anal., 21 (1990), pp. 823836.

[6] J. F. BABADJiAn AND M. BAÍA, Multiscale nonconvex relaxation and application to thin films, Asymptot. Anal., 48 (2006), pp. 173-218.

[7] H. T. Banks, V. A. Bokil, D. Cioranescu, N. L. Gibson, G. Griso, and B. Miara, Homogenization of periodically varying coefficients in electromagnetic materials, J. Sci. Comput., 28 (2006), pp. 191-221.

[8] M. BARCHIESI, Multiscale homogenization of convex functionals with discontinuous integrand, J. Convex Anal., 14 (2007), pp. 205-226. 
[9] G. Bayada, S. Martin, and C. Vazquez, Two-scale homogenization of a hydrodynamic Elrod-Adams model, Asymptot. Anal., 44 (2005), pp. 75-110.

[10] G. BAyada, S. Martin, ANd C. VAzQuez, Homogenization of a nonlocal elastohydrodynamic lubrication problem: A new free boundary model, Math. Models Methods Appl. Sci., 15 (2005), pp. 1923-1956.

[11] A. Bensoussan, J. L. Lions, and G. Papanicolaou, Asymptotic Analysis for Periodic Structures, Stud. Math. Appl. 5, North-Holland, Amsterdam, 1978.

[12] D. Blanchard, A. Gaudiello, and G. Griso, Junction of a periodic family of elastic rods with a $3 d$ plate, Part I, J. Math. Pures Appl., 88 (2007), pp. 1-33.

[13] D. Blanchard, A. Gaudiello, and G. Griso, Junction of a periodic family of elastic rods with a thin plate, Part II, J. Math. Pures Appl., 88 (2007), pp. 149-190.

[14] D. Blanchard And G. Griso, Microscopic effects in the homogenization of the junction of rods and a thin plate, Asymptot. Anal., 56 (2008), pp. 1-36.

[15] A. Bossavit, G. Griso, and B. Miara, Modelling of periodic electromagnetic structures: Bianisotropic materials with memory effects, J. Math. Pures Appl., 84 (2005), pp. 819 850.

[16] A. Bourgeat, S. Luckhaus, and A. Mikelic, Convergence of the homogenization process for a double-porosity model of immiscible two-phase flow, SIAM. J. Math. Anal., 27 (1996), pp. 1520-1543.

[17] A. Braides and A. Defranceschi, Homogenization of Multiple Integrals, Oxford Lect. Ser. Math. Appl. 12, Oxford University Press, Oxford, 1998.

[18] A. Braides and D. LukKassen, Reiterated homogenization of integral functionals, Math. Models Methods Appl. Sci., 10 (2000), pp. 1-25.

[19] D. A. G. BRugGeman, Berechnung verschiedener physikalisher konstanten von heterogenen substanzen, Ann. Physik., 24 (1935), p. 634.

[20] J. CAsado-Díaz, Two-scale convergence for nonlinear Dirichlet problems in perforated domains, Proc. Roy. Soc. Edinburgh Sect. A, 130 (2000), pp. 249-276.

[21] J. Casado-Díaz And M. Luna-Laynez, A multiscale method to the homogenization of elastic thin reticulated structures, in Homogenization 2001, GAKUTO Internat. Ser. Math. Sci. Appl. 18, Gakkōtosho, Tokyo, 2003, pp. 155-168.

[22] J. Casado-Díaz, M. Luna-Laynez, and J. D. Martín, An adaptation of the multi-scale methods for the analysis of very thin reticulated structures, C.R. Acad. Sci. Paris, Ser. 1, 332 (2001), pp. 223-228.

[23] J. Casado-Díaz, M. Luna-Laynez, and J. D. Martín, A new approach to the analysis of thin reticulated structures, in Homogenization 2001, GAKUTO Internat. Ser. Math. Sci. Appl. 18, Gakkōtosho, Tokyo, 2003, pp. 257-262.

[24] D. Cioranescu, A. Damlamian, and G. Griso, Periodic unfolding and homogenization, C.R. Acad. Sci. Paris, Ser. 1, 335 (2002), pp. 99-104.

[25] D. Cioranescu, A. Damlamian, and G. Griso, The Stokes problem in perforated domains by the periodic unfolding method, in Proceedings of the Conference on New Trends in Continuum Mechanics, M. Suliciu, ed., Theta, Bucarest, 2005, pp. 67-80.

[26] D. Cioranescu, A. Damlamian, G. Griso, and D. Onofrei, The periodic unfolding method for perforated domains and Neumann sieve models, J. Math. Pures Appl., 89 (2008), pp. 248-277.

[27] D. Cioranescu, A. Damlamian, and R. De Arcangelis, Homogenization of nonlinear integrals via the periodic unfolding method, C.R. Acad. Sci. Paris, Ser. 1, 339 (2005), pp. $77-82$.

[28] D. Cioranescu, A. Damlamian, And R. De Arcangelis, Homogenization of integrals with pointwise gradient constraints via the periodic unfolding method, Ricerche Mat., 55 (2006), pp. 31-54.

[29] D. Cioranescu, A. Damlamian, and R. De Arcangelis, Homogenization of quasiconvex integrals via the periodic unfolding method, SIAM J. Math. Anal., 37 (2006), pp. 14351453.

[30] D. Cioranescu and P. Donato, An Introduction to Homogenization, Oxford Lecture Ser. in Math. Appl. 17, Oxford University Press, Oxford, 1999.

[31] D. Cioranescu, P. Donato, and R. Zaki, The periodic unfolding method in perforated domains, Port. Math., 63 (2006), pp. 467-496.

[32] D. Cioranescu, P. Donato, And R. Zaki, Asymptotic behavior of elliptic problems in perforated domains with nonlinear boundary conditions, Asymptot. Anal., 53 (2007), pp. 209-235.

[33] G. Dal Maso and A. Defranceschi, Correctors for the homogenization of monotone operators, Differential Integral Equations, 3 (1990), pp. 1151-1166. 
[34] A. Damlamian, An elementary introduction to periodic unfolding, in Proceedings of the Narvik Conference 2004, GAKUTO Internat. Ser. Math. Sci. Appl. 24, A. Damlamian, D. Lukkassen, A. Meidell, and A. Piatnitski, eds., Gakkōtosho, Tokyo, 2006, pp. 119-136.

[35] A. Damlamian and P. Donato, $H^{0}$-convergence and iterated homogenization, Asymptot. Anal., 39 (2004), pp. 45-60.

[36] A. Damlamian and K. Pettersson, Homogenization of oscillating boundaries, Discrete Contin. Dyn. Syst., 23 (2009), pp. 197-219.

[37] A. Damlamian, N. Meunier, and J. Van Schaftingen, Periodic homogenization of monotone multivalued operators, Nonlinear Anal., 67 (2007), pp. 3217-3239.

[38] A. Ene and J. Saint Jean Paulin, On a model of fractured porous media, in Mathematical Modelling of Flow Through Porous Media, A. Bourgeat, C. Carasso, S. Luckhaus, and A. Mikelic, eds., World Scientific, River Edge, NJ, 1995, pp. 402-409.

[39] M. Ghergu, G. Griso, H. Mechkour, and B. Miara, Homogenization of thin piezoelectric shells, ESAIM: M2AN Math. Modeling Numer. Anal., 41 (2007), pp. 875-895.

[40] G. GRISO, Analyse asymptotique de structures réticulées, Thèse Université Pierre et Marie Curie (Paris VI), Paris, 1996.

[41] G. GRIso, Thin reticulated structures, in Progress in Partial Differential Equations, The Metz Surveys 3, M. Chipot, J. Saint Jean Paulin, and I. Shafrir, eds., Pitman, London, 1994, pp. 161-182.

[42] G. GRISO, Error estimate and unfolding for periodic homogenization, Asymptot. Anal., 40 (2004), pp. 269-286.

[43] G. GRISO, Les méthodes d'éclatement en homogénéisation périodique et en élasticité linéarisée, Thèse d'Habilitation, Université Pierre et Marie Curie, Paris, 2005.

[44] G. GRIso, Interior error estimates for periodic homogenization, C.R. Acad. Sci. Paris, Ser. 1, 340 (2005), pp. 251-254.

[45] G. GRISO, Interior error estimates for periodic homogenization, Anal. Appl., 4 (2006), pp. 6179.

[46] G. Griso and E. Rohan, On homogenization of diffusion-deformation problem in strongly heterogeneous media, Ricerche Mat., 56 (2007), pp. 161-188.

[47] G. Griso And T. Sanchez-Rua, Homogenization of an elasticity problem for a catalyst support by using the unfolding method, submitted.

[48] M. Lenczner, Homogénéisation d'un circuit électrique, C.R. Acad. Sci. Paris, Ser. 2, 324 (1997), pp. 537-542.

[49] M. LenCZner AND D. Mercier, Homogenization of periodic electrical networks including voltage to current amplifiers, SIAM Multiscale Model. Simul., 2 (2004), pp. 359-397.

[50] M. Lenczner and G. Senouci-Bereksi, Homogenization of electrical networks including voltage-to-voltage amplifiers, Math. Models Methods Appl. Sci., 9 (1999), pp. 899-932.

[51] M. Lenczner, M. Kader, And P. Perrier, Modèle à deux échelles de l'équation des ondes à coefficients oscillants, C.R. Acad. Sci. Paris, Ser. 1, 328 (2000), pp. 335-340.

[52] J. L. Lions, D. Lukkassen, L. E. Persson, and P. Wall, Reiterated homogenization of monotone operators, Chinese Ann. Math. Ser. B, 22 (2001), pp. 1-12.

[53] D. LukKassen, Reiterated homogenization of non-standard Lagrangians, C.R. Acad. Sci. Paris, Ser. 1, 332 (2001), pp. 999-1004.

[54] D. LukKassen and G. W. Milton, On hierarchical structures and reiterated homogenization, in Proceedings of the Conference on Function Spaces, Interpolation Theory and Related Topics in Honor of Jack Peetre on his 65th Birthday, M. Cwikwl, M. Engliš, A. Kufner, L.-E. Persson, and G. Sparr, eds, Walter de Gruyter, Berlin, 2002, pp. 311-324.

[55] D. Lukkassen, G. Nguetseng, and P. Wall, Two-scale convergence, Int. J. Pure Appl. Math., 2 (2002), pp. 35-86.

[56] N. Meunier and J. Van Schaftingen, Periodic reiterated homogenization for elliptic functions, J. Math. Pures Appl., 9 (2005), pp. 1716-1743.

[57] G. Milton, The Theory of Composites, Cambridge University Press, Cambridge, 2002.

[58] G. NGuetseng, A general convergence result for a functional related to the theory of homogenization, SIAM J. Math. Anal., 20 (1989), pp. 608-629.

[59] O. A. Oleinik, A. S. Shamaev, and G. A. Yosifian, Mathematical Problems in Elasticity and Homogenization, North-Holland, Amsterdam, 1992.

[60] D. OnOFREI, The unfolding operator near a hyperplane and its applications to the Neumann sieve model, Adv. Math. Sci. Appl., 16 (2006), pp. 239-258. 